ESAIM: M2AN

Vol. 41, No 2, 2007, pp. 391-426

DOI: $10.1051 / \mathrm{m} 2 \mathrm{an}: 2007018$
ESAIM: Mathematical Modelling and Numerical Analysis

www.edpsciences.org/m2an

\title{
ATOMISTIC TO CONTINUUM LIMITS FOR COMPUTATIONAL MATERIALS SCIENCE
}

\author{
Xavier Blanc ${ }^{1}$, Claude Le Bris ${ }^{2,3}$ and Pierre-Louis Lions ${ }^{4,5}$
}

\begin{abstract}
The present article is an overview of some mathematical results, which provide elements of rigorous basis for some multiscale computations in materials science. The emphasis is laid upon atomistic to continuum limits for crystalline materials. Various mathematical approaches are addressed. The setting is stationary. The relation to existing techniques used in the engineering literature is investigated.
\end{abstract}

Mathematics Subject Classification. 35-xx, 39-xx, 41-xx, 49-xx, 65-xx, 68-04, 73-xx.

Received November 30, 2005.

\section{INTRODUCTION AND OUTLINE}

Recent years have witnessed a considerable development of numerical simulation techniques coupling models at different scales, see e.g. the monographs [3,14,44,59,85,117,121] and also [103] for most recent developments. Such methods, generically termed multiscale methods, are increasingly used for the simulation of matter, in fields as diverse as computational chemistry, biology, materials science.

The extensive use of such methods legitimately raises the question of their mathematical foundation. In addition to this theoretical concern, the related questions of numerical analysis of the techniques, and, further, improvement of their efficiency, need to be addressed.

Placing the multiscale simulations on a firm mathematical ground is an ambitious goal. It is an overwhelming task considering the variety of methods, and the scope of multiscale simulations. It is therefore mandatory to restrict one's ambition. A field of predilection for such a mathematical enterprise is the multiscale simulation of solid materials (and in fact mostly crystalline materials). It turns out that the periodic nature of crystalline materials is a favorable feature for the mathematical setting (see $[6,7,87]$.

\footnotetext{
Keywords and phrases. Problems of mechanics, variational problems, discrete to continuum limit, multiscale models, homogenization theory, $\Gamma$-limit, quasiconvexity, gradient flows, quasicontinuum method, adaptivity.

${ }^{1}$ Laboratoire J.-L. Lions, Université Pierre et Marie Curie, Boîte courrier 187, 75252 Paris, France. blanc@ann.jussieu.fr

2 CERMICS, École Nationale des Ponts et Chaussées, 6-8 avenue Blaise Pascal, Cité Descartes, 77455 Marne-la-Vallée.

3 MICMAC, Inria Rocquencourt, 78153 Le Chesnay, France. lebris@cermics.enpc.fr

4 Collège de France, 11 place Marcelin Berthelot, 75231 Paris Cedex 05, France.

5 CEREMADE, Université Paris Dauphine, place du Maréchal de Lattre de Tassigny, 75775 Paris Cedex 16, France.

lions@ceremade.dauphine.fr
} 
But even with this restriction, the scope of the methods remain incredibly large, as exemplified by all the existing variants of methods proceeding from the homogenization paradigm. See [16] for a seminal contribution to the field. Homogenization, both in its historical analytical form, and in its more recent numerical form, can indeed be considered as the ancestor of all recent multiscale methods, and is still a very active field for research. However, for the multiscale simulation of solid materials, a new field has recently emerged. Although somehow based on the homogenization concept, it follows a bottom line significantly different from homogenization methods. This field consists of the family of methods coupling an atomistic description and a continuous description of matter. The models and techniques that can be considered as members of this family are very large. An abundant literature testifies to the growing practical importance of this approach: $[2,3,44-47,56,59,62-68,85,86,88,94,99-105,107,117,122-126,132-134,138]$.

Very impressive simulations indeed provide useful qualitative information, and satisfactory quantitative results are often obtained. Although the methods are functioning well, there is only a poor mathematical understanding, and the opportunities for improvement are significant. No systematic assessment of the quality is available, and the mathematically inclined reader will acknowledge with us that this can be considered as a dangerous feature. It does not exist either any rationale for improving the efficiency of the numerical techniques. In summary, huge efforts are needed to bridge the gap between the existing numerical simulations and the state of the art of the mathematical knowledge. Concentrating on multiscale models used for the simulation of crystalline materials that couple an atomistic and a continuous description of matter, the present article reviews some attempts to bridge this gap. The derivation of the models will be primarily addressed. The last section of this article addresses some other issues.

To some extent, the present article can be seen as the sequel of a previous work [53]. That publication was dedicated to a state-of-the-art review of the mathematical foundations of the models of computational chemistry, both for molecules and for crystals. The focus was entirely on the purely microscopic scale at the atomistic level. In contrast, and as a follow-up, the present article investigates the micro-to-macro limit of the microscopic models addressed in [53]. It shows how the theoretical issues in such a limit process are actually a first step toward the rigorous justification of the derivation of the multiscale models in use in computational materials science. A second step would consist of an overview of the mathematical analysis of the multiscale model itself, hopefully followed by a numerical analysis of the approximation techniques in use, in the vein of what was performed in [93] for the microscopic models of computational chemistry. Unfortunately, the subject of the mathematical and numerical analysis of such multiscale models is still mostly unexplored (apart from some preliminary studies that will be mentioned below). When possible, some elements for the analysis of the models will be given. This is however only a limited part of the present work (Sect. 3). We anticipate a huge development of the multiscale methods in the years to come and look forward to a more complete and substantial review when there is a sufficient material.

This article describes various tracks followed by groups of mathematicians to address the issues mentioned above. The exposition is biased toward the work on the three authors. However, as often as possible considering the space limitations, some incursions into the work of other groups will be made, in particular with a view to comparing the viewpoints, and demonstrating how they complement one another. Sections 2.2 and 3.3 have this purpose.

It is important to note that we exclusively consider the stationary setting. Of course, time-dependent multiscale simulations do exist, and are very important. Their mathematical understanding is also a challenge, based upon different techniques, which we will not address here. To some extent, the stationary setting is much better understood.

We begin by motivating, in one among many possible ways, the use of multiscale models for the numerical simulation in materials science. We find it indeed important to give the reader a flavor of the challenging difficulties faced by practitioners of the field, and the way the multiscale methods allow these difficulties to be overcome, circumvented, or at least conveniently treated. 


\subsection{Motivation}

Let us first briefly recall the standard mechanical description of a material subjected to forces (see e.g. [55] for a detailed mathematically oriented presentation). For pedagogic purposes and for brevity of the exposition, the setting is static, but analogous considerations, often more complicated though, could be developed in a time-dependent setting.

We denote by $\mathcal{D}$ the reference domain ${ }^{1}$ the material occupies at rest, by $\varphi$ the deformation it is subjected to, i.e. the map from $\mathcal{D}$ to $\mathbb{R}^{3}$ that gives the current position of the material. We also denote by $u(x)=\varphi(x)-x$ the displacement, and by

$$
F=\nabla \varphi: \mathcal{D} \longrightarrow \mathcal{M}_{3},
$$

the gradient of deformation, where $\mathcal{M}_{3}$ denotes the space of square matrices of size $3 \times 3$.

The general equations that describe the equilibrium of our sample material, when subjected to body forces $f$ and boundary forces $g$ read

$$
\left\{\begin{array}{rll}
-\operatorname{div} T & =f, & \text { in } \mathcal{D} \\
T \cdot n & =g, & \text { on } \partial \mathcal{D} .
\end{array}\right.
$$

Here, $T$ denotes the stress tensor (more precisely the first Piola-Kirchhoff stress tensor), and $n$ is the unitary outward normal vector on $\partial \mathcal{D}$.

In order to close the equations (1.1), a relation must be postulated between the stress tensor $T$ and the kinematic description of the material, provided by the fields $\varphi, u$ or $F$. In contrast to equations (1.1) which are general, the relation linking $T$ to, say, $\varphi$, depends on the material considered. In such a relation is indeed encoded the physical and mechanical nature of the material. Formally, such a closure relation reads

$$
T=T(x, \varphi(x), \ldots)
$$

and is called a constitutive relation, or a law of behavior. Equation (1.2) is symbolic: derivatives of $\varphi$ may also be inserted, as well as other points than $x$ (or, in a time-dependent setting, times prior than the time $t$ at which the stress tensor is evaluated). The relation may even be a differential equation, a partial differential equation, an integral equation, etc.

A usual assumption in deriving such a law is the elasticity of the material:

$$
T=T(x, F(x))
$$

Hyperelasticity is often additionally assumed: the material is assumed to dissipate no energy during a cyclic deformation. From this is inferred the existence of a density of mechanical energy, i.e. a function $W$ such that

$$
T(F)=\frac{\partial W}{\partial F}(F) .
$$

Actually, owing to the fact that the laws of mechanics need to be invariant under rigid rotations, the function $W$ may only depend on $F$ via $F^{t} F$. Along with (1.4), equation (1.1) is then recognized as the Euler-Lagrange equations for the minimization of a problem of the type

$$
\inf _{\varphi \in \mathcal{A}} \int_{\mathcal{D}} W(\nabla \varphi(x)) \mathrm{d} x-\int_{\mathcal{D}} f \varphi-\int_{\partial \mathcal{D}} g \varphi
$$

where $\mathcal{A}$ is the set of all admissible deformations $\varphi$ compatible with the possible boundary conditions (we do not make precise here the somewhat delicate definition of admissible deformations, and refer to the bibliography

\footnotetext{
${ }^{1}$ In the present article, we call domain of $\mathbb{R}^{d}$ any bounded, open, connected, subset of $\mathbb{R}^{d}$.
} 
$[10,55])$. The most famous example, although of course oversimplified, is the model of linearized elasticity: it is considered that the tensor of deformation

$$
e=\frac{1}{2}\left({ }^{t} F F-1\right)=\frac{1}{2}\left(\nabla u+{ }^{t} \nabla u+{ }^{t} \nabla u \cdot \nabla u\right)
$$

may be satisfactorily approximated by its linearized version $\varepsilon=\frac{1}{2}\left(\nabla u+{ }^{t} \nabla u\right)$, and that the stress tensor simply reads $T=A: \varepsilon$, where $A$ is a fixed fourth order symmetric tensor. The corresponding density of mechanical energy reads as the simple quadratic form

$$
W=\frac{1}{2} \varepsilon: A: \varepsilon .
$$

The derivation of the constitutive law (1.2), or equivalently in the hyperelasticity setting, that of the density (1.4) of mechanical energy $W$, is a central theoretical question, and challenging practical issue faced by mechanics. Then, the resolution of (1.1) (coupled with (1.3)), or the minimization of (1.5), is the purpose of the numerical simulations performed. Hundreds of men/years of efforts have been devoted to the improvements of both the constitutive laws (1.2) and the techniques used to simulate them.

Despite all these efforts and all the expertise accumulated, the strategy for the derivation of a constitutive law has, at least, two major flaws.

First, even when the derivation of the law itself is feasible (and it is often not the case, as will be seen below), it is extremely difficult to evaluate the impact of a possibly imperfect constitutive law on the final result of the simulations. Assessing the validity of a constitutive law is as crucial a subject as deriving the law. This must be borne in mind. The sequel will present a means to envision a competitive modelling strategy, useful (at least) for the sake of comparison.

Second, and as importantly, there are a number of situations when the determination of a meaningful constitutive equation is an overwhelming task. First, the constitutive laws are typically determined in the following manner: an analytic form is derived using a priori theoretical considerations on the nature of the material at hand, and then the parameters (such as multiplicative constants or exponents in the power laws) are determined using a fitting procedure with experimental data on test beds. Such test situations cannot reproduce the whole generality of situations encountered in practice. For instance, the most traditional materials, the behavior of which is very well known in usual situations, are sometimes subsequently considered in unusual conditions. The latter may be quite far from the ideal conditions in the laboratory, used for the derivation of the constitutive law. This occurs, in particular, when the assumptions of continuum mechanics are not valid. The constitutive law is thus by definition invalid also. An instance is the initiation of a crack in a material: in the vicinity of the crack, it is clear that the assumptions of continuum mechanics do not hold. Likewise, a constitutive law is typically valid for a perfect material, and it is not clear how the phenomena related to fatigue can be rigorously accounted for in such a setting. In this respect, extreme cases consist of situations not accessible to experiment, such as those encountered when determining the behavior of matter under extremely high pressures (like considering a material sample in the earth mantel), or after extremely long times (like predicting the 30 -year old damage of materials caused by radiation in nuclear plants). In addition, new materials, with new properties, are engineered on a daily basis, and the experimental determination of their constitutive law may be a too long procedure. Further, some new materials may be envisioned to enjoy specific properties, for which an estimation of the behavior needs to be determined before synthesis. Clearly, the standard approach has to be complemented.

It follows from the above considerations that it is useful to bypass the derivation of a constitutive law. This is one of the major purpose of the multiscale models of materials science. In principle, the constitutive law is expected to encode the physical nature of the material, and translate it, analytically, in a relation between the stress and the strain, so that the phenomena taking place at all the scales in the material finer than the macroscopic ones are implicitly accounted for. A multiscale model is also aimed at encoding such a relation, but, in contrast, without translating it into an explicit mathematical relation. The physical nature of the material is inserted via an explicit microscopic description, which is in turn coupled with the usual macroscopic 
description of the material. Loosely speaking, the derivation of the constitutive law is implicitly performed by the simulation itself. The amount of physical intuition needed is expected to be smaller, the computational effort will compensate for it. Likewise, it is hoped that less modelling assumptions will be needed: ideally, a universal microscopic model is inserted in the universal macroscopic description (1.1). In doing so, the sources for inaccuracies are easier to identify, and the a posteriori assessment of the quality of the result is simpler.

Of course, the use of a more systematic model comes at a price: the computational cost of a multiscale model is orders of magnitude larger than that of a usual "purely macroscopic" model. In the present state of the art, the former cannot compete with the latter on real scale computations. However, three arguments help to advocate such multiscale methods. First, they are often very well adapted to a parallel numerical resolution. Second they can serve as a numerical test-bed: a few costly multiscale computations can be performed on "samples", in the back room, so as to determine more efficiently than usually an adequate constitutive law, which in turn will be used in practical real-scale simulations. Third, and this last point is somehow related to the purpose of this article, the multiscale models have not benefited from the hundreds of men/years of efforts, we were refereeing to above and the usual macroscopic approaches have benefited from. It is not forbidden to think that extensive efforts by applied mathematicians in the field will both improve the quality of the methods, and reduce their computational cost.

\subsection{Set-up of the problem}

In all the above discussion, the terminology microscopic/macroscopic is to be understood in a broad sense, without any reference to a particular quantitative length scale. For the sequel, as mentioned earlier, the microscopic model we will consider is an atomistic one, at the scale of the nanometer.

With this restriction, let us give some substance to the general discussion above. We put ourselves in the variational setting (1.5), and discuss a simple example of multiscale model.

Assuming that the material is a perfect (periodic) crystal, we model its energy at the microscopic scale by a simple pair potential model. We fix some interaction potential $V$, assume that the atomic sites fill in some ideal periodic lattice $\mathcal{L}$ in the reference configuration, so that, when subjected to a deformation $\varphi$, the energy of the material reads

$$
\frac{1}{2} \sum_{\overline{x_{i}} \in \mathcal{L}} \sum_{\overline{x_{j}} \neq \overline{x_{j}} \in \mathcal{L}} V\left(\varphi\left(\overline{x_{i}}\right)-\varphi\left(\overline{x_{j}}\right)\right),
$$

where the factor one-half aims at avoiding double-counting. To some extent, the above energy is the exact energy of the material deformed by $\varphi$, since it accounts for all the interactions between the atomic sites of the material. Note however that this is a controversy issue, that we shall not consider here. The point is of course that such a double sum is impossible to compute in practice, because of the number of atomic sites involved, say of the order of the Avogadro number $10^{23}$. Fortunately, in most situations evaluating the sum (1.6) is also useless since the state of the material deformed by $\varphi$ (and, for simplicity, subjected to no force) can be satisfactorily modeled in the framework of hyperelasticity, using a density of mechanical energy $W$, by the integral

$$
\int_{\mathcal{D}} W(\nabla \varphi(x)) \mathrm{d} x
$$

See Remark 2.1 below for a related discussion.

Combining the two expressions (1.6) and (1.7) is thus a natural idea in order to build a multiscale model. Roughly, the argument goes as follows. Consider a deformation $\varphi$ applied to the material. In regions of the material where the deformation is "regular" (in a somewhat vague sense, varies at a scale much larger than the microscopic one), the derivation of a convenient macroscopic density of mechanical energy is assumed to hold true, and the energy of the deformed material is expressed by an integral of the form (1.7). Let us denote the union of such regions by $\mathcal{D}_{\text {reg }}$. On the other hand, some parts of the material, forming the set $\mathcal{D}_{\text {sing }}=\mathcal{D} \backslash \mathcal{D}_{\text {reg }}$, may be subjected to a "nasty" deformation $\varphi$ (say with singularities, oscillations, etc., at a scale comparable to 
the microscopic one). There, the best to do is to appeal to the original atomistic model (1.6) for the expression of the energy. Schematically again, the energy of the entire material is then given by

$$
\int_{\mathcal{D}_{\text {reg }}} W(\nabla \varphi(x)) \mathrm{d} x+\frac{1}{2} \sum_{\overline{x_{i}} \in \mathcal{L} \cap \mathcal{D}_{\text {sing }}} \sum_{\overline{x_{j}} \neq \overline{x_{j}} \in \mathcal{L} \cap \mathcal{D}_{\text {sing }}} V\left(\varphi\left(\overline{x_{i}}\right)-\varphi\left(\overline{x_{j}}\right)\right) .
$$

Formula (1.8) is the prototypical expression of the energy of a deformed configuration in a multiscale model coupling a continuum description with an atomistic one. Just to name one method among others, let us mention the quasicontinuum method, from [88,106,107,125,126,132-134], that more or less involves energy functionals of the form (1.8).

Many variants of model (1.8) exist. More sophisticated descriptions are possible, both at the continuum level and at the atomistic level. We will see some of them below. The spirit of the modelling however remains the same, and for most of our formal arguments, the form (1.8) is a convenient object to study.

A number of theoretical issues, relevant to practice, follow from the consideration of the energy (1.8).

The most important one is to understand how the density of mechanical energy $W$ is related to the pair potential interaction $V$. Indeed, it is a matter of consistency that when employed over a region where the deformation $\varphi$ is regular, the atomistic model (1.6) provides an energy that is close to the integral form (1.7). Depending on the viewpoint chosen, the atomistic model (1.6) should be conveniently approximated, in the limit of a regular deformation, by the continuous model (1.7), or, alternatively, the continuous model (1.7) must be a macroscopic limit of the discrete model (1.6).

A second important issue is a question of adaptivity. Indeed, the region $\mathcal{D}_{\text {sing }}$ is conveniently chosen so as to embed all the singularities of the deformation $\varphi$. On the other hand, with a view to reducing as much as possible the computational cost, this region must be kept as small as possible. So there is an interest in a systematic analysis of the choice of the regions $\mathcal{D}_{\text {reg }}$ and $\mathcal{D}_{\text {sing }}$, depending on the deformation $\varphi$. This is all the more true as, one should not forget this, the deformation $\varphi$ is not given in advance, but is an unknown of the problem.

On top of that, there is the key issue of determining the deformation $\varphi$ that gives the state of the material in the situation considered. In the variational setting, $\varphi$ is the minimizer of the energy (1.8), possibly with additional terms accounting for the forces applied to the material, and subject to appropriate constraints. Thus a minimization problem of the type (1.5), posed with the energy functional (1.8), is considered. The question arises as to know whether the deformation $\varphi$ to be considered in practice is the global minimizer of such a problem, or only one of its critical points (which one?). The question is also to design a numerical strategy so as to determine this $\varphi$.

The purpose of this article is to review the mathematical understanding on such questions, for various settings. Section 2 deals with the problem of deriving a macroscopic hyperelastic energy from the atomic level. The question of adaptivity is briefly discussed in Section 3.2, and Sections 3.1 and 3.3 deal with possible ways to determine the deformation $\varphi$.

\section{Stationary SETTING: DERIVATION OF MACROSCOPIC ENERGIES}

In the present section, we investigate the relation between the microscopic model (exemplified in the energy functional (1.8) by the pair potential $V$ ) and the macroscopic model (i.e. in (1.8) the density of mechanical energy $W$ ). We will mainly follow two different tracks. In Section 2.1, we consider that the deformation $\varphi$ is a common object, both seen by the macroscopic level and the microscopic level. We have implicitly assumed this when writing formula (1.8). In Section 2.2, we will use another approach, based on the $\Gamma$-limit paradigm. Then, the deformation the atomic sites experience is part of the problem in the passage from the microscale to the macroscale. In other words, the atomic sites do not really see the deformation imposed at the macroscopic level, but experience a rearrangement, in order to minimize the energy (see for instance (2.51) and (2.52) below). 


\subsection{Pointwise approaches}

Our purpose here is the following:

- we give ourselves a microscopic description of the sample (in the simplest possible case, it is a pair potential energy between the sites of a periodic lattice);

- we fix the deformation $\varphi$ that is imposed to the material at the macroscopic level and directly apply it to the atomic sites at the microscopic level;

- we search for the macroscopic limit of the energy obtained.

Proceeding so, we expect to derive an explicit form for the macroscopic mechanical energy of the sample deformed by $\varphi$, thus a link between the density of mechanical energy $W$, and, say, the pair potential $V$ used at the microscopic level.

In a second step, in Sections 2.1.2 and 2.1.3 respectively, we will complexify the description at the microscopic level, considering microstructures less simple than a perfect periodic lattice, and energy functionals more sophisticated than the simple pair-potential.

\subsubsection{The simplest possible microscopic setting}

Let us look at the material at the microscopic scale. It occupies the domain $\mathcal{D}$, and we assume its substructure is the truncation $\mathcal{D} \cap \mathcal{L}$ of a periodic lattice $\mathcal{L}$, whose sites $\overline{x_{i}} \in \mathcal{D} \cap \mathcal{L}$ interact with a pair potential $V$. For clarity of exposition, we assume the periodic lattice has a cubic unit cell, and that the site stands at the center of this cell. The length of the cell is first fixed to unity, so that the lattice $\mathcal{L}$ is indeed $\mathbb{Z}^{3}$. However, the length will soon be denoted by $\varepsilon$, a quantity that will go to zero in the macroscopic limit. The potential $V$, taken radially symmetric, is assumed to be smooth, and have compact support. No body force, nor boundary force, is applied to the material. The deformation we will apply to the sample is assumed to be smooth. All this aims at avoiding unnecessary technicalities.

We proceed in two steps. First, we have to understand what the energy of this assembly of sites is, at rest, i.e. for a deformation $\varphi$ that is Identity. In the present simple setting, this first step is straightforward, but it is important, with a view to generality, to still identify it.

Obviously, the energy of the material reads

$$
\frac{1}{2} \sum_{\overline{x_{i}} \in \mathcal{D} \cap \mathbb{Z}^{3}} \sum_{\overline{x_{j}} \neq \overline{x_{i}} \in \mathcal{D} \cap \mathbb{Z}^{3}} V\left(\overline{x_{i}}-\overline{x_{j}}\right) .
$$

As we already pointed out above, the number of sites in $\mathcal{D} \cap \mathbb{Z}^{3}$ is a huge number, typically of the order of the Avogadro number. Thus, a natural idea is to search for the limit of this quantity when the number of sites goes to infinity, i.e. when $\mathcal{D}=\mathbb{R}^{3}$ and the lattice is the whole lattice $\mathbb{Z}^{3}$. Obviously, one cannot expect (2.1) to have a finite limit in this regime. This is justified: the energy is an extensive quantity, and only the energy per unit volume, or per unit particle, can be expected to have a meaning. Therefore the right question to ask is the existence of the limit

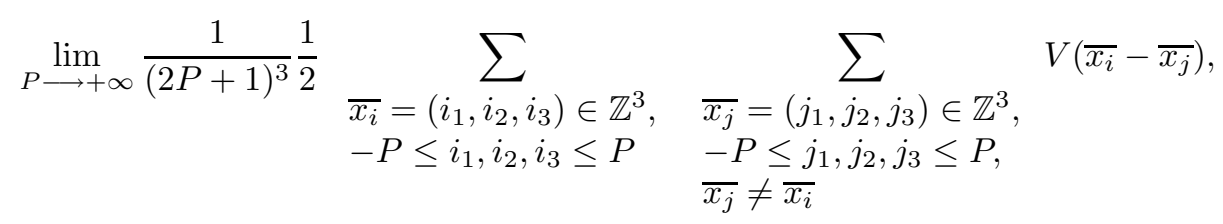

where it has been assumed that $\mathcal{D}$ was a cubic box of (large) size $P$, for simplicity. The number of sites per dimension is $N=2 P+1$. It is then a simple exercise to see that this limit is

$$
\frac{1}{2} \sum_{\overline{x_{k}} \in \mathbb{Z}^{3}, \overline{x_{k}} \neq 0} V\left(\overline{x_{k}}\right) .
$$


This concludes our first step, which is the determination of a quantity that can be identified as the energy of the microscopic periodic lattice. This has been performed through a limit process indeed known as the thermodynamic limit process: considering a system of finite size (here the truncation of a periodic lattice), we have let the size (and in fact the volume) of the system go to infinity, and searched for the limit of its energy per unit volume. This limit process is one of the basic tools of statistical mechanics. The terminology thermodynamic limit is somewhat misleading, since there is no temperature effect involved here (the models we manipulate are at zero temperature). A more proper denomination would be bulk limit, but we employ the word thermodynamic limit to follow the literature, see for instance [98]. The thermodynamic limit for a periodic lattice interacting via a pair potential performed here is an easy issue. The argument above clearly extends to a more general pair potential $V$, that does not need to be smooth, nor compactly supported. Basically what is needed is that the formula (2.3) makes sense, which is the case when $V$ is continuous and $V \in L^{1}\left(\mathbb{R}^{3}\right)$. The sequel will present the adaptation for more general settings.

A point to note for the sequel is that, should the cubic lattice $\mathbb{Z}^{3}$ be replaced by a general lattice $A \mathbb{Z}^{3}$ (where $A$ is a fixed symmetric $3 \times 3$ matrix), the energy then reads

$$
\frac{1}{2} \sum_{\overline{x_{k}} \in \mathbb{Z}^{3}, \overline{x_{k}} \neq 0} V\left(A \overline{x_{k}}\right)
$$

For our second step, let us now apply the deformation $\varphi$ and go back to the expression (2.1), which now reads, as (1.6):

$$
\frac{1}{2} \sum_{\overline{x_{i}} \in \mathcal{D} \cap \mathbb{Z}^{3}} \sum_{\overline{x_{j}} \neq \overline{x_{i}} \in \mathcal{D} \cap \mathbb{Z}^{3}} V\left(\varphi\left(\overline{x_{i}}\right)-\varphi\left(\overline{x_{j}}\right)\right) .
$$

We intend to investigate the macroscopic limit of the energy (2.5). To this end, we first need to reintroduce the small length scale $\varepsilon$ in the problem: the sites of the lattice now span the set $\mathcal{D} \cap(\varepsilon \mathbb{Z})^{3}$. Correspondingly, we need to rescale the potential $V$ by a factor $\varepsilon$, so that its equilibrium length (i.e., say, the length $r$ that minimizes the function $r \longrightarrow V(r)$ ) should be also of order $\varepsilon$ for consistency. Therefore, we overall modify (2.5) into

$$
\frac{1}{2} \sum_{\overline{x_{i}} \in \mathcal{D} \cap \mathbb{Z}^{3}} \sum_{\overline{x_{j}} \neq \overline{x_{i}} \in \mathcal{D} \cap \mathbb{Z}^{3}} V\left(\frac{\varphi\left(\varepsilon \overline{x_{i}}\right)-\varphi\left(\varepsilon \overline{x_{j}}\right)}{\varepsilon}\right) .
$$

Considering as above that $\mathcal{D}$ is a large cube, replacing $\varepsilon$ by $\frac{1}{N}=\frac{1}{2 P+1}$ (so that the sample overall occupies a unit length along each dimension), and renormalizing the sum so as to get the energy per unit particle, we obtain the formula analogous to (2.6):

$$
\begin{array}{cl}
\frac{1}{(2 P+1)^{3}} & \sum^{\overline{x_{i}}=\left(i_{1}, i_{2}, i_{3}\right) \in \mathbb{Z}^{3},} \\
-P \leq i_{1}, i_{2}, i_{3} \leq P & \begin{array}{l}
\overline{x_{j}}=\left(j_{1}, j_{2}, j_{3}\right) \in \mathbb{Z}^{3} \\
-P \leq j_{1}, j_{2}, j_{3} \leq P \\
\overline{x_{j}} \neq \overline{x_{i}}
\end{array}
\end{array}
$$

The purpose of the second step of our argument is to identify the limit of $(2.7)$ when $N \longrightarrow \infty$. This means that we both let the truncated lattice go to the whole infinite lattice at the microscopic scale, and let the length of the lattice go to zero, so as to pass to the macroscopic limit. Again, in this simple case, the analysis is obvious. As $\varphi$ is smooth, we write its Taylor expansion at the first order and neglect the higher order terms:

$$
\frac{\varphi\left(\frac{\overline{x_{i}}}{N}\right)-\varphi\left(\frac{\overline{x_{j}}}{N}\right)}{\frac{1}{N}}=N\left(\varphi\left(\frac{\overline{x_{i}}}{N}\right)-\varphi\left(\frac{\overline{x_{j}}}{N}\right)\right) \approx \nabla \varphi\left(\frac{\overline{x_{j}}}{N}\right) \cdot\left(\overline{x_{i}}-\overline{x_{j}}\right),
$$


insert this in the potential $V$, and in turn search for the first order. Arguing formally, omitting some technicalities in particular related to boundary terms that can be easily handled, and using the periodicity of the lattice, we see that the limit to be determined reads

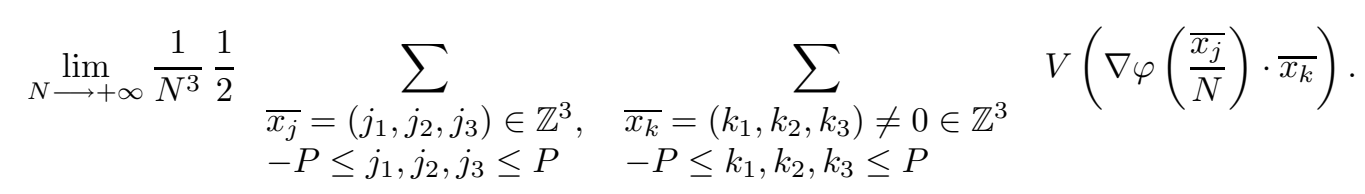

Denoting by

$$
\Psi(x)=\frac{1}{2} \sum_{\bar{x}_{k} \neq 0 \in \mathbb{Z}^{3}} V\left(\nabla \varphi(x) \cdot \overline{x_{k}}\right),
$$

we observe that $(2.9)$ is of the form

$$
\lim _{\operatorname{card}\left\{y_{j}\right\} \rightarrow+\infty} \frac{1}{\operatorname{card}\left\{y_{j}\right\}} \sum_{y_{j}} \Psi\left(y_{j}\right),
$$

where the points $y_{j}$ form a grid in a unit volume, and thus is a Riemann sum, which converges as $N \longrightarrow+\infty$ to

$$
\frac{1}{2} \int_{\mathcal{D}} \sum_{\overline{x_{k}} \neq 0 \in \mathbb{Z}^{3}} V\left(\nabla \varphi(x) \cdot \overline{x_{k}}\right) \mathrm{d} x .
$$

At the macroscopic level, our density of mechanical energy therefore reads

$$
W(\nabla \varphi(x))=\frac{1}{2} \sum_{\overline{x_{k}} \neq 0 \in \mathbb{Z}^{3}} V\left(\nabla \varphi(x) \cdot \overline{x_{k}}\right) .
$$

Comparing with (2.3), and more precisely with its extension (2.4), we observe that this energy is indeed the energy of the original periodic lattice deformed by the linear map $\nabla \varphi(x)$ at the macroscopic point $x$. It can be easily checked that if $V$ is radially symmetric, the right-hand side of (2.11) actually depends only on $F^{t} F$, and not $F=\nabla \varphi(x)$.

For the simple case treated here (periodic lattice, pair-potential interaction), this derivation of a macroscopic density of energy was certainly known for long, and part of the folklore of the mathematical analysis of the models of mechanics. The work [30], announced and summarized in [29], however presents a rigorous and systematic study of such a question, in a variety of settings. It must be borne in mind that the regularity of the deformation $\varphi$ plays a role in the derivation. It is easily understandable, since the above argument uses a Taylor expansion. In fact, the deformation is requested to be a diffeomorphism, i.e.

$$
\exists a, b>0, \quad \forall x, y \in \mathcal{D}, \quad a|x-y| \leq|\varphi(x)-\varphi(y)| \leq b|x-y|
$$

and to be sufficiently regular. All this is made precise in [30]. Conditions less stringent than the original ones were then given in [26]. The sequel will provide more details on the work [30], and other studies by other groups in the same vein $[1,4,36-41,67,71,73,75,76,113,122-124]$.

In a somewhat provocative manner, we could say that we may conclude here this section on the derivation of macroscopic energies. Indeed, formula (2.3) giving the energy of the lattice at the microscopic scale, and formula (2.11) expressing the macroscopic density of energy that follows, suffice to give a foundation to the prototypical multiscale model (1.8). All other cases are corollaries, or variants of these two results. However, the large variety of models existing motivates further explanations. Therefore, in Sections 2.1.2 and 2.1.3, we now explain how the same two steps, leading respectively to the energy (2.3) and to the density of mechanical 
energy (2.11), may be performed for more general settings than the present one. Actually, we will obtain that the formula generalizing $(2.11)$ reads

$$
W(\nabla \varphi(x))=\text { Energy of the microstructure at macro point } x \text { deformed by } \nabla \varphi(x) .
$$

The mutiscale nature of the model is obvious on that formula.

In addition, the technique we have used above to compute the density (2.11) is not the only existing one. There are other manners to proceed, making different assumptions, and using different approaches. We will thus mention in Section 2.2 some other approaches, by other groups of researchers.

Remark 2.1. The strategy we have described so far may also be seen as a way to explicitly compute the energy at the microscopic level. Indeed, consider that we model the energy of the material under study at the microscopic scale, i.e. at the atomic scale, using a formula of the form (2.7), which may also be written as

$$
E_{N}^{\varphi}=\frac{1}{N^{3}} \frac{1}{2} \sum_{\overline{x_{i}} \in A_{N}} \sum_{\overline{x_{j}} \neq \overline{x_{i}} \in A_{N},} V\left(\frac{\varphi\left(\overline{x_{i}}\right)-\varphi\left(\overline{x_{j}}\right)}{\varepsilon}\right),
$$

where $A_{N}$ is a subset of $(\varepsilon \mathbb{Z})^{3}$ such that $\# A_{N} \approx N^{3}$. In practice, this number is of the order of the Avogadro number $\left(10^{23}\right)$, and such a sum is not tractable. However, using the method we have presented, we see that if $\varepsilon$ is of order $1 / N$, then (2.14) is a Riemann sum approximating the integral (2.10). Computing this integral with an adapted discretisation will thus give a good approximation of (2.10)

A variant of the above approach is presented in [4], along with the analysis of some other possible schemes for deriving continuum energies from the atomistic level. The observation that motivates this variant, called inner expansion technique and advocated in [4] is the following. In the above approach, called the scaling approach in [4], the scaling $\frac{1}{N}$ plays two roles at the same time. It is the interatomic distance, that aims to go to zero in the thermodynamic limit process. It also gives the space step that is used for the Riemann sum, approaching in the limit the continuous integral giving the macroscopic energy. This is obvious in the expression $V\left(\nabla \varphi\left(\frac{\overline{x_{j}}}{N}\right) \cdot \overline{x_{k}}\right)$. In fact, the two length scales need not coincide. A possibility is to keep the interatomic distance as fixed, say $h$, and choose some other length $\tilde{h}$ for the space step for approximating the discrete sum by an integral. In contrast to $h$, which is fixed, $\tilde{h}$ aims to go to zero. In spirit, such an approach is related to the quasicontinuum technique (see $[88,106,107,125,126,132-134]$ ) where so-called representative atoms (separated from distances at the scale $\tilde{h}$ ) are chosen to expand the interatomic interaction. In particular, the number of sites is kept finite, since $h$ is fixed. In this setting, a Taylor expansion of the deformation at any site of the lattice is performed, in function of the deformation at the representative points (i.e. the grid at the scale $\tilde{h}$ ). In the limit $\tilde{h} \longrightarrow 0$, densities of macroscopic energy are obtained, which may differ from those obtained by the above technique. Just to give an idea of the method, let us consider a one-dimensional setting and assume the pair interaction potential is a nearest neighbor interaction, i.e. an atomistic energy of the form

$$
\sum_{\overline{x_{i}}} V\left(\overline{x_{i+1}}-\overline{x_{i}}\right)
$$

In other words, there is only the terms $j=i+1$ and $j=i-1$ in the sum (2.1). Applying the deformation $\varphi$ yields

$$
\sum_{\overline{x_{i}}} V\left(\varphi\left(\overline{x_{i+1}}\right)-\varphi\left(\overline{x_{i}}\right)\right) .
$$

If it is now assumed that $\overline{x_{i+1}}-\overline{x_{i}}=h$ is fixed, and we introduce the representative point

$$
y_{i}=\frac{1}{2}\left(\overline{x_{i}}+\overline{x_{i+1}}\right),
$$


then the Taylor expansion of $\varphi$ implies that

$$
V\left(\varphi\left(\overline{x_{i+1}}\right)-\varphi\left(\overline{x_{i}}\right)\right)=V\left(\nabla \varphi\left(y_{i}\right) \cdot h+\frac{1}{24} \nabla^{3} \varphi\left(y_{i}\right) h^{3}+\text { h.o.t. }\right)
$$

Next, one considers that the distance $y_{i+1}-y_{i}$ (in fact equal to $h$ ) is a scale $\tilde{h}$ which goes to zero, asymptotically. Therefore the density of macroscopic energy obtained is

$$
V\left(\nabla \varphi(x) \cdot h+\frac{1}{24} \nabla^{3} \varphi(x) h^{3}+\text { h.o.t. }\right)
$$

If the Taylor expansion is truncated at the first order, it coincides with (2.11), which in the particular case of a nearest neighbor interaction (2.15) indeed reads $V(\nabla \varphi(x))$. Notice that the scale $h$, kept fixed here, is in fact to equal to the unit length, implicitly set to unity in the above approach. On the other hand, if the third order Taylor expansion is performed, or higher orders expansion, then the result differs from (2.11). The work [4] presents an analysis of the method, and useful comparisons with others. The work interestingly complements the literature.

\subsubsection{More general models}

The preceding section presents the bottom line for the derivation of the density of the mechanical energy $W$ from the microscopic model, and when the latter is a simple pair potential model, when the macroscopic deformation is directly applied to the atomic sites. This is far from covering the variety of models commonly used at the microscopic scale in materials science. Still assuming that the macroscopic deformation is applied to the microscopic scale, we now expose how the preceding study can be extended to more general models.

It is standard to consider that the atomic sites of the lattice are occupied by nuclei, the latter being equipped with an electronic cloud. This electronic cloud consists of electrons that are modeled with a quantum model. In materials science, the most popular models are models from Density Functional Theory, although some wave functions models, such as Hartree-Fock and post Hartree-Fock type models may also be employed. We now investigate to what extent the derivation of Section 2.1.1 applies to these two categories of models, respectively.

Before we turn to this, let us simply mention that for further details on such models from computational quantum chemistry, we refer e.g. to the monographs $[20,77,116,131]$ in the chemistry literature, along with the monograph [92] and the state-of-the-art review articles [53,93] in the mathematical literature.

The mathematical understanding of the models from Density Functional Theory relies very much upon the understanding of ancestors of these models, namely the Thomas-Fermi type models [97,98,127]. This is why the monograph [50] (whose results were priorly summarized in [48]) is devoted to the thermodynamic limit problem for such models. This work is concerned with Thomas-Fermi-von Weizsäcker type models, and is an extension of the previous very important works [98] on the Thomas-Fermi model. Landmark references addressing the same type of questions include works by L. Van Hove, D. Ruelle, or Ch. Fefferman.

As mentioned in the previous section, the first step of the derivation of the density $W$ consists of the determination of the thermodynamic limit. In a model such as the Thomas-Fermi-von Weizsäcker model, the question is to determine a model for the energy of a perfect crystal. If we follow the line mentioned in Section 2.1.1, the setting is clear. We consider a truncation of the lattice $\mathcal{L}$ that we denote by $\mathcal{L}_{N}$ and counts $N^{3}$ atomic sites, say each of them occupied by a nucleus with unit charge (for simplicity). We equip these $N^{3}$ nuclei with $N^{3}$ electrons. We write the energy of such a truncated lattice: it is the energy of interaction between the nuclei, plus the contribution from the electronic structure. Formally, even if there is no real chemical compound corresponding to this mathematical construction, the truncated lattice can be considered as a molecule, and then we approach the full lattice by a sequence of such molecules, letting the nuclei fill in the whole lattice, and, jointly, letting the number of electrons grow. In mathematical terms, and in the specific 
context of the Thomas-Fermi-von Weizsäcker model, this means we consider the ground state energy

$$
I_{N}^{T F W}=\inf \left\{E_{T F W}(\rho), \quad \rho \geq 0, \quad \sqrt{\rho} \in H^{1}\left(\mathbb{R}^{3}\right), \quad \int_{\mathbb{R}^{3}} \rho=N^{3}\right\}
$$

where

$$
\begin{aligned}
E_{T F W}(\rho)= & C_{W} \int_{\mathbb{R}^{3}}|\nabla \sqrt{\rho}|^{2}+C_{T F} \int_{\mathbb{R}^{3}} \rho^{5 / 3} \\
& -\sum_{\overline{x_{k}} \in \mathcal{L}_{N}} \int_{\mathbb{R}^{3}} \frac{\rho(x)}{\left|x-\overline{x_{k}}\right|}+\frac{1}{2} \int_{\mathbb{R}^{3}} \int_{\mathbb{R}^{3}} \frac{\rho(x) \rho(y)}{|x-y|} \mathrm{d} x \mathrm{~d} y+\frac{1}{2} \sum_{\bar{x}_{j} \neq \overline{x_{k}} \in \mathcal{L}_{N}} \frac{1}{\left|\overline{x_{j}}-\overline{x_{k}}\right|}
\end{aligned}
$$

is the energy functional, and ask for the existence of the limit of the energy per unit particle (or per unit volume) $\frac{I_{N}^{T F W}}{N^{3}}$. In fact, a related question is to ask whether the electronic density $\rho_{N}$ that minimizes $I_{N}^{T F W}$ for each $N$ indeed goes to a limit as $N$ goes to infinity and whether this limit is periodic. In other words, the question is: does the electronic cloud reproduce in the limit the invariance of the distribution of nuclei, i.e. the periodicity.

The latter question on the density is much more difficult than the former on the energy. However it turns out that for the Thomas-Fermi type models, the most elegant and general way to answer to the former question is to first answer to the second.

For the TFW model, [50] presents a proof of the convergence (up to an explicit additive universal constant) of $\frac{I_{N}^{T F W}}{N^{3}}$ to the periodic ground state energy

$$
\begin{gathered}
I_{\text {per }}^{T F W}\left(\mathbb{Z}^{3}\right)=\inf \left\{E_{\text {per }}^{T F W}(\rho) ; \rho \geq 0, \sqrt{\rho} \in H_{\text {per }}^{1}(Q), \int_{Q} \rho=1\right\}, \\
E_{\text {per }}^{T F W}(\rho)=C_{W} \int_{Q}|\nabla \sqrt{\rho}|^{2}+C_{T F} \int_{Q} \rho^{5 / 3}-\int_{Q} \rho(x) G(x) \mathrm{d} x+\frac{1}{2} \iint_{Q \times Q} \rho(x) \rho(y) G(x-y) \mathrm{d} x \mathrm{~d} y,
\end{gathered}
$$

together with the convergence of $\rho_{N}$ to the minimizer $\rho_{\text {per }}$ of (2.18). In the above formula, $Q$ is the unit cell of the periodic lattice, $H_{p e r}^{1}(Q)$ is the subset of $H_{l o c}^{1}\left(\mathbb{R}^{3}\right)$ consisting of functions which satisfy the periodic boundary conditions on the boundary of $Q$ and the potential $G$ which appears in the definition (2.19) of the TFW functional is defined, in a unique way, by

$$
-\Delta G=4 \pi\left(-1+\sum_{y \in \mathbb{Z}^{3}} \delta(\cdot-y)\right), \quad \int_{Q} G=0, \quad G \text { is periodic. }
$$

The similarity of the periodic problem (2.18)-(2.19) with the molecular problem (2.16)-(2.17) is to be emphasized. The forms of the energy functionals (2.19) and (2.17) are identical ${ }^{2}$, except for two features: integrals on the unit cell $Q$ replace integrals on the space $\mathbb{R}^{3}$, and the periodic potential $G$, Green function of the Laplace operator on $Q$, replaces the Coulombic potential $\frac{1}{|x|}$, Green function of the Laplace operator on $\mathbb{R}^{3}$. In physical terms, the configuration space is periodic and no longer extends to infinity, and the interaction potential accounts for the periodicity as well. We will see again such a similarity in the subsequent sections, for other settings.

\footnotetext{
${ }^{2}$ Note for the reader not familiar with such models: some easy manipulations are needed to really observe this similarity for the electrostatic terms, we refer to [50] for the details.
} 
It is important to understand that such a result is not straightforward. Indeed, it can be easily checked that a priori each of the last three terms of the electrostatic energy involved in $(2.17)$

$$
-\sum_{\overline{x_{k}} \in \mathcal{L}_{N}} \int_{\mathbb{R}^{3}} \frac{\rho(x)}{\left|x-\overline{x_{k}}\right|} \mathrm{d} x+\frac{1}{2} \iint_{\mathbb{R}^{3} \times \mathbb{R}^{3}} \frac{\rho(x) \rho(y)}{|x-y|} \mathrm{d} x \mathrm{~d} y+\frac{1}{2} \sum_{\overline{x_{j}} \neq \overline{x_{k}} \in \mathcal{L}_{N}} \frac{1}{\left|\overline{x_{j}}-\overline{x_{k}}\right|}
$$

scales in $N^{5}$. Therefore, cancelation effects are needed to obtain an energy scaling linearly with respect to the volume $N^{3}$. A key point is therefore to prove that the electronic density spreads in an homogeneous way so that the electrostatic interaction between nuclei is screened by the electronic cloud, thereby making possible the appropriate scaling law for the energy.

As mentioned above, the more efficient strategy for proving the convergence of the energy is indeed to investigate the limit of the electronic density $\rho_{N}$. For each $N, u_{N}=\sqrt{\rho_{N}}$ is the solution to the system

$$
\left\{\begin{array}{l}
-C_{W} \Delta u_{N}+\frac{5}{3} C_{T F} u_{N}^{7 / 3}-\Phi_{N} u_{N}=0 \\
u_{N} \geq 0 \\
-\frac{1}{4 \pi} \Delta \Phi_{N}=\sum_{\overline{x_{k}} \in \mathcal{L}_{N}} \delta\left(\cdot-\overline{x_{k}}\right)-\rho_{N}
\end{array}\right.
$$

where $\Phi_{N}$ is called the effective potential.

Some a priori bounds proved on $\rho_{N}$ and $\Phi_{N}$ allow to pass to the limit in the Euler Lagrange system (2.22) and obtain the system

$$
\left\{\begin{array}{l}
-C_{W} \Delta u+\frac{5}{3} C_{T F} u^{7 / 3}-\Phi u=0 \\
u \geq 0 \\
-\frac{1}{4 \pi} \Delta \Phi=m-\rho
\end{array}\right.
$$

for the pointwise limits $(u, \Phi)$ of $\left(u_{N}, \Phi_{N}\right)$. The main result that will allow to conclude is a result of unique solvability of system (2.23) in appropriate functional spaces, provided a convenient assumption is made on $m$. This assumption as a particular case of the periodicity of $m$ and reads: $m$ is a locally bounded measure that satisfies

$$
\left\{\begin{array}{l}
\sup _{x \in \mathbb{R}^{3}} m\left(x+B_{1}\right)<+\infty \\
\exists R, \inf _{x \in \mathbb{R}^{3}} m\left(x+B_{R}\right) \geq 1
\end{array}\right.
$$

These conditions are more or less known as those defining a Delaunay lattice in crystallography [60]. Heuristically, assumptions (2.24) exclude situations where the set of nuclei include infinitely many charged clusters of nuclei somewhere or infinitely huge empty zones.

Under this assumption, standard, but intricate, arguments of the theory of nonlinear elliptic PDEs allow to establish unique solvability (see [50] for the details, and [15,43] for related results). A key ingredient in the proof is the strict convexity of the TFW energy functional $(2.17)$ with respect to $\rho=u^{2}$. This strict convexity translates into appropriate properties of the system (2.23). When uniqueness is established, a simple argument of translation shows that if $m$ is periodic, which is the case here, then $\rho$ and $\Phi$ also are periodic. The limit $\rho$ is then recognized as $\rho_{\text {per }}$, the minimizer of (2.18). Consequently, the average energy (here the periodic energy) is then found by simply inserting the convergence of $u_{N}$ and $\Phi_{N}$ in all terms of the energy functional. The thermodynamic limit is thus entirely determined. Step 1 of the argument of Section 2.1.1 is thus achieved, and we may claim that $I_{\text {per }}^{T F W}$ as defined by $(2.18)$ is a proper definition for the energy of the periodic lattice $\mathbb{Z}^{3}$ in the setting of the TFW model. It generalizes the definition (2.3) for the pair interaction potential $V$. Of course, if the lattice $\mathbb{Z}^{3}$ is replaced by $A \mathbb{Z}^{3}$, then the cell $Q$ in (2.18) is to be replaced by $A Q$ and the potential $G$ by the corresponding one $G_{A}$. 
Let us now proceed to the second step of our derivation, namely the micro-to-macro limit. We know from the argument of Section 2.1.1 that, the deformation $\varphi$ being fixed, this derivation is only a rewriting of the thermodynamic limit process, along with a change of scale. Here again, treating the problem via the EulerLagrange equation is the convenient approach.

Consider the Euler-Lagrange equation (2.23), that we reproduce here for convenience, expliciting the measure $m$ :

$$
\left\{\begin{array}{l}
-C_{W} \Delta u+\frac{5}{3} C_{T F} u^{7 / 3}-\Phi u=0, \\
u \geq 0 \\
-\frac{1}{4 \pi} \Delta \Phi=\sum_{\overline{x_{i}} \in \mathbb{Z}^{3}} \delta\left(\cdot-\overline{x_{i}}\right)-\rho .
\end{array}\right.
$$

We perform the following three modifications on it. First, we apply the deformation $\varphi$ to the sites $\overline{x_{i}}$ of $\mathcal{L}$, so that the sites are now $\varphi\left(\overline{x_{i}}\right)$. Second, we translate from some $\varphi\left(\overline{x_{j}}\right)$ for some index $j$ such that $\frac{\overline{x_{j}}}{N}$ converges to the macroscopic point $x$ in the limit. Third, we rescale all the inter-atomic distances. These three modifications result in the system

$$
\left\{\begin{array}{l}
-C_{W} \Delta u+\frac{5}{3} C_{T F} u^{7 / 3}-\Phi u=0, \\
u \geq 0 \\
-\frac{1}{4 \pi} \Delta \Phi=\sum_{\overline{x_{i}} \in \mathbb{Z}^{3}} \delta\left(\cdot-\frac{\varphi\left(\frac{\overline{x_{i}}}{N}\right)-\varphi\left(\frac{\overline{x_{j}}}{N}\right)}{\frac{1}{N}}\right)-\rho .
\end{array}\right.
$$

These manipulations are exactly analogous to those leading from $V\left(\overline{x_{i}}-\overline{x_{j}}\right)$ to $V\left(\frac{\varphi\left(\frac{\overline{x_{i}}}{N}\right)-\varphi\left(\frac{\overline{x_{j}}}{N}\right)}{\frac{1}{N}}\right)$ in Section 2.1.1. Notice that, because of the deformation, the sites do not form a periodic lattice. Thus the measure inserted in $(2.25)$ is not a periodic measure any longer, but, for $\varphi$ convenient, this system nevertheless admits a unique solution, owing to the fact that

$$
m_{j}=\sum_{\overline{x_{i}} \in \mathbb{Z}^{3}} \delta\left(\cdot-\frac{\varphi\left(\frac{\overline{x_{i}}}{N}\right)-\varphi\left(\frac{\overline{x_{j}}}{N}\right)}{\frac{1}{N}}\right)
$$

enjoys the convenient properties mentioned above.

The sequel of the argument again mimics that of Section 2.1.1. For $N$ large, the measure (2.26) may be approximated by

$$
m_{j}=\sum_{\overline{x_{i}} \in \mathbb{Z}^{3}} \delta\left(\cdot-\nabla \varphi\left(\frac{\overline{x_{j}}}{N}\right)\left(\overline{x_{i}}-\overline{x_{j}}\right)\right)
$$

and one recovers in the right-hand side a sum of Dirac masses standing at sites of a periodic lattice. System (2.25) is thus, approximately, the Euler-Lagrange equation associated to the TFW energy of the lattice $\nabla \varphi\left(\frac{\overline{x_{j}}}{N}\right) \mathbb{Z}^{3}$, i.e. (2.18) with $Q$ replaced by $\nabla \varphi\left(\frac{\overline{x_{j}}}{N}\right) Q$. Also for $N$ large, we may formally replace $\frac{\overline{x_{j}}}{N}$ by the generic macroscopic point $x$, as in a Riemann sum. This formal argument leads to the macroscopic energy

$$
\int_{\mathcal{D}} I_{\text {per }}^{T F W}\left(\nabla \varphi(x) \mathbb{Z}^{3}\right) \mathrm{d} x
$$

The density of mechanical energy obtained therefore reads

$$
W(\nabla \varphi(x))=I_{p e r}^{T F W}\left(\nabla \varphi(x) \mathbb{Z}^{3}\right)
$$

where $I_{\text {per }}^{T F W}\left(\nabla \varphi(x) \mathbb{Z}^{3}\right)$ is defined as in (2.18). We observe this is of the general form (2.13). Of course, we have only outlined the proof. The above lines need to be made rigorous, and this is performed in [30]. There is however no fundamental difficulty in doing so, and we will not bother the reader with unnecessary technicalities. 
This concludes our study of the TFW case, that we see here as a prototypical example of quantum model for the microscopic scale.

It is worth emphasizing that

(a) the thermodynamic limit process is the key step in the determination of the micro-to-macro limit allowing for the derivation of the density of mechanical energy $W$; the second step only amounts to a Taylor expansion;

(b) the fundamental ingredient of the proof of the thermodynamic limit is the result of uniqueness stated above for the limit of the Euler-Lagrange equation; recovering the limit of the energy is then a simple matter;

(c) this uniqueness result holds because the underlying energy functional (here the TFW energy functional $(2.17))$ is indeed strictly convex with respect to the density $\rho$; more sophisticated models involving non convex energy functionals cannot be addressed; the argument was not detailed here for the sake of brevity, but it is obvious in [50] that the strict convexity plays an instrumental role.

In order to show that, indeed, the situation is not always as "simple" as that for the TFW model, let us turn to the Hartree-Fock model. Hartree-Fock type models are indeed a case of interest for which the thermodynamic limit is not a closed problem, but where some partial results are available. Let us briefly mention them. Together with Hartree-type models, which will not at all be reviewed here, these are the subjects of the works $[51,52]$, summarized in [49].

The Hartree-Fock ground-state energy is defined by

$$
I_{\mathcal{L}_{N}}^{H F}=\inf \left\{E_{\mathcal{L}_{N}}^{H F}(\gamma) ; \gamma \in \Gamma_{N}\right\}
$$

where the Hartree-Fock functional, expressed in terms of density matrices $\gamma$, reads

$$
E_{\mathcal{L}_{N}}^{H F}(\gamma)=\operatorname{Tr}[-\Delta \gamma]+E_{\mathcal{L}_{N}}^{\text {elec }}(\gamma(x, x))-\frac{1}{2} \iint_{\mathbb{R}^{3} \times \mathbb{R}^{3}} \frac{|\gamma(x, y)|^{2}}{|x-y|} \mathrm{d} x \mathrm{~d} y
$$

with $E_{\mathcal{L}_{N}}^{\text {elec }}(\gamma(x, x))$ defined by $(2.21)$. In the above expression of the energy,

$$
\operatorname{Tr}[-\Delta \gamma]=\sum_{n \geq 1} \lambda_{n} \int_{\mathbb{R}^{3}}\left|\nabla \varphi_{n}(x)\right|^{2} \mathrm{~d} x
$$

and the set of admissible density matrices $\Gamma_{N}$ is composed of density matrices satisfying in addition $\gamma^{2}=\gamma$, $\operatorname{Tr}(\gamma)=N$ and that $\operatorname{Tr}[-\Delta \gamma]<+\infty$. Recall that by definition a density matrix is a self-adjoint operator on $L^{2}\left(\mathbb{R}^{3}\right)$, with finite trace, such that

$$
0 \leq \gamma \leq \mathbf{1}
$$

(in the sense of self-adjoint operators) where $\mathbf{1}$ denotes the identity on $L^{2}\left(\mathbb{R}^{3}\right)$, and whose trace fits the number of electrons. A density matrix being a Hilbert-Schmidt operator on $L^{2}\left(\mathbb{R}^{3}\right)$, its kernel may be decomposed along a complete set of orthonormal eigenfunctions $\left(\varphi_{n}\right)_{n \geq 1} \in L^{2}\left(\mathbb{R}^{3}\right)$ of $\gamma$ associated to the eigenvalues $0 \leq \lambda_{n} \leq 1$, in such a way that $\gamma(x, y)=\sum_{n \geq 1} \lambda_{n} \varphi_{n}(x) \varphi_{n}^{\star}(y)$.

There is no result to date that rigorously establishes the thermodynamic limit of the HF problem. As above, this owes to the fact that the HF model is not convex. Nevertheless, there is such a result for the restricted Hartree-Fock model, which consists in omitting the exchange term in the HF model, i.e. the last term of (2.28). The analysis is then far simpler because convexity is restored. This model can be shown to converge to the 
RHF periodic model

$$
E_{\text {per }}^{R H F}\left(\gamma_{\text {per }}\right)=\frac{1}{(2 \pi)^{3}} \int_{Q^{\star}} \operatorname{Tr}_{L_{\xi}^{2}(Q)}\left[-\Delta_{\xi} \gamma_{\xi}\right] \mathrm{d} \xi-\int_{Q} G \rho_{\text {per }}+\frac{1}{2} \iint_{Q \times Q} \rho_{\text {per }}(x) G(x-y) \rho_{\text {per }}(y) \mathrm{d} x \mathrm{~d} y
$$

in the thermodynamic limit where $Q^{\star}$ is the unit cell of the dual lattice of $\mathbb{Z}^{3}$, and $\gamma_{\xi}$ is the Bloch decomposition of $\gamma$ (see $[51,52]$ for the details.

On the basis of this, and also considering other partial results established on specific variants of the Hartree model (see [52]), it is natural to postulate that the Hartree-Fock energy per unit volume converges to the following periodic model:

$$
\begin{gathered}
I_{\text {per }}^{H F}=\inf \left\{E_{\text {per }}^{H F}\left(\gamma_{p e r}\right) ; \gamma_{\text {per }} \in \Gamma\right\} \\
E_{\text {per }}^{H F}\left(\gamma_{p e r}\right)=\frac{1}{(2 \pi)^{3}} \int_{Q^{\star}} \operatorname{Tr}_{L_{\xi}^{2}(Q)}\left[-\Delta_{\xi} \gamma_{\xi}\right] \mathrm{d} \xi-\int_{Q} G \rho_{p e r} \\
+\frac{1}{2} \iint_{Q \times Q} \rho_{\text {per }}(x) G(x-y) \rho_{\text {per }}(y) \mathrm{d} x \mathrm{~d} y-\frac{1}{2} \iint_{Q \times \mathbb{R}^{3}} \frac{\left|\gamma_{p e r}(x, y)\right|^{2}}{|x-y|} \mathrm{d} x \mathrm{~d} y .
\end{gathered}
$$

This model can be shown to be well posed and admits a minimizer. The Euler-Lagrange equation satisfied by any minimizer are indeed the equations solved in the numerical simulations (see the details in [51]). Using these equations, and rescaling the data conveniently as we did in the TFW case, a micro-to-macro limit can be performed (we did not go through all the detailed proof, though). Unfortunately, the argument is not complete since the thermodynamic limit is only postulated.

To conclude this section, let us emphasize the following fact. Most models of practical interest arising from the DFT theory, or from wave function methods (as HF-type models) are non convex. E.g., a very popular DFT-type model such as, say, a Kohn-Sham model with a local density approximation for the correlation energy, is not convex. Therefore, for most of these models of practical interest, we are not able to perform the derivation of the density of mechanical energy, since we are not even able to perform the thermodynamic limit and define the energy of the crystalline phase at the microscopic scale, which is the first step of our program. In the absence of such a rigorous derivation, it can be conjectured that an expression of the type (2.13), the energy being defined by a ground state periodic energy such as (2.18) or (2.29), remains valid. This is clearly a theoretical concern, and a track for further research.

\subsubsection{More general geometries}

The derivation of a macroscopic density of energy, performed above in the pair potential case and extended to some quantum models, is not restricted to a periodic geometry of atomic sites. As mentioned above, the two ingredients are the thermodynamic limit and a Taylor expansion. So in any situation where both tools can be used, the program may be fulfilled.

Straightforward extensions. The proof of the thermodynamic limit, for the pair potential, the TFW model, and any convenient model, readily extends to geometries close to periodic. We mean e.g. quasiperiodic structure such as those of quasicrystals, see the details in [50].

Likewise, structures periodic in one or two dimensions, embedded in the 3-dimensional physical space can be treated. This is the case of structures, periodic along one direction and compact in the two other directions, somewhat in the spirit of long linear molecules such as polymers. This is also the case for thin films, i.e. a structure periodic in 2 dimensions and consisting of a few rows of atoms in the third direction. The derivation of the microscopic energy of both cases was performed in [24]. Such situations obviously do not satisfy the assumptions (2.24) on the measure $m$. In both the above situations, these assumptions are nevertheless fulfilled "along the direction of" the infinite part of the system, which is the only necessary property needed. 
Two remarks are in order. First, the derivation was restricted to the case of one-dimensional structures along straight lines, and planar two dimensional structures. The adaptation to curved structures may be more intricate (see [5] for a work in this direction). Second, the micro-to-macro limit was not performed in [24], but the ingredients of [30], combined with those of [24], should suffice to derive the macroscopic density of energy. We however did not check this in details.

With a view to treating geometries more general than periodic, and also for the purpose of getting closer to the modelling of real materials, an extension of the setting concerns grains. Real solid materials are indeed often aggregates of grains, each grain consisting of a single perfect crystal (or something more complicated than that, but let us stick to this case for clarity of exposition). Although we did not perform the proof in details for this case, we believe that our arguments of the periodic case allow to derive a macroscopic density of the type

$$
\begin{aligned}
W(\nabla \varphi(x))= & \begin{array}{l}
\text { Energy of the microstructure at macro point } x \text { deformed by } \nabla \varphi(x), \\
\text { weighted by the probability of this microstructure. }
\end{array}
\end{aligned}
$$

An explicit example of such a density is

$$
W(\nabla \varphi(x))=\int I_{p e r}^{T F W}\left(\nabla \varphi(x) A \mathbb{Z}^{3}\right) \Psi(A, x) \mathrm{d} A
$$

where $\Psi(A, x)$ denotes the density of probability to find at the macroscopic point $x$ a grain consisting of a periodic crystal $A \mathbb{Z}^{3}$. The integral above spans all the possible matrices $A$ defining all the periodic crystals. For simplicity, we omit some technical details related to the labeling of periodic structures. We only give here the spirit of the modelling.

An extension in another direction is the following. In the argument of Section 2.1.1, it is obvious that the Taylor expansion could be pushed to the second order, and even higher orders. Proceeding so, one may derive densities of energies accounting not only for the bulk energy but also for the energy of the outer surface of the crystal. We refer to [30] for the details. The idea of specifically defining the energy of the surface of a crystal was pursued in [22]. The work focuses on the notion of a microscopic energy. The derivation of a macroscopic energy, in a sense to be defined, was not investigated.

Intimately related to the question of the energy of surfaces is the question of the energy of interfaces. This case has a particular twofold interest. First, it is very important from the application viewpoint. Second, if one applies a deformation to a bicrystal, i.e. a structure consisting of two different crystals glued to one another via an interface, there is no point in believing that the deformation will be regular when crossing the interface. Therefore, to be consistent, the mathematical setting is thus different from that introduced in Section 2.1.1. Typically, we apply a deformation $\varphi$ for which the gradient $\nabla \varphi$ contains a singularity at the interface. Loosely speaking, the interesting mathematical phenomenon is concentrated along the normal direction to the interface, assumed planar for simplicity. The study thus reduces to a one-dimensional study, in a simple setting at least. This motivates our study [26] of a one-dimensional situation. For brevity, let us only state our result. We consider two different one-dimensional periodic structures, say $A$ and $B$, separated by another one-dimensional structure, a joint $C$ centered at the origin. We model the energy by a pair potential interaction: $V$ is the interaction potential between any two sites belonging to $A$ and $B, V_{1}$ is the interaction potential between two sites when one of them belongs to $C$. We pass to the macroscopic limit when the width $\gamma$ of the joint $C$ goes to zero, and correspondingly the interatomic distance goes to zero (much faster than $\gamma$ ). Two regimes are investigated, depending on whether we rescale differently or not the interaction $V$ and $V_{1}$. This is for the purpose of modelling a rigid joint or a soft joint between the two crystals. In the limit, two macroscopic energies are obtained (here $\mathcal{D}=A \cup B$ )

$$
E(u)=\frac{1}{2|\mathcal{D}|} \int_{A} \sum_{k \in \mathbb{Z} \backslash\{0\}} V\left(\varphi^{\prime}(x) k\right)+\frac{1}{2|\mathcal{D}|} \int_{B} \sum_{k \in \mathbb{Z} \backslash\{0\}} V\left(\varphi^{\prime}(x) k\right)+\frac{\gamma}{|\mathcal{D}|} V_{1}\left(\left[\varphi^{\prime}\right](0)\right)+o(\gamma)
$$


in the case of a rigid joint, and

$$
E(u)=\frac{1}{2|\mathcal{D}|} \int_{A} \sum_{k \in \mathbb{Z} \backslash\{0\}} V\left(\varphi^{\prime}(x) k\right)+\frac{1}{2|\mathcal{D}|} \int_{B} \sum_{k \in \mathbb{Z} \backslash\{0\}} V\left(\varphi^{\prime}(x) k\right)+\frac{1}{|\mathcal{D}|} V_{1}\left(\left[\varphi^{\prime}\right](0)\right)
$$

in the case of a soft joint. Here, $\left[\varphi^{\prime}\right](0)$ is the jump of $\varphi^{\prime}$ at 0 .

Both (2.32) and (2.33) agree with models well known in the mechanics literature [75, 76,95,96, 140], and obtained by an homogenization procedure performed purely at the macroscopic scale.

Fully non periodic structures. The previous extensions are close to periodic structures, or concern simple rearrangements of periodic structures.

However, it is interesting to address situations where the geometrical structure is far from being periodic. At least three reasons could motivate considering such cases. First, there are sample of matters that cannot be considered to have a periodic structure: think to a material with many fractures, that consists of many different materials, at different scales, etc. Second, derivating a macroscopic energy requires applying a macroscopic deformation which, even when the original structure is periodic, may lead to the consideration of fully non periodic structures. Third, all our work so far has dealt with models at zero temperature. This is a first step. But the full understanding of the mechanical behavior of a sample of matter requires accounting for temperature effects (see Sect. 3.3 below for a related discussion). Now a periodic structure may vary much under the influence of temperature and reach truly non periodic configurations.

Let us add to these three situations a purely theoretical concern. In fact, even if the experimental observation confirms that matter at zero temperature is most often periodic (we exclude exotic situations in this simplified exposition), it is not proved that the models used reproduce this property. In physics, the terminology employed for the theoretical study of this question is crystal problem: suppose we give ourselves a system of $N$ identical nuclei of unit charge and provide them with $N$ electrons, suppose we let the positions of the nuclei relax to their most stable positions, the electrons relaxing also in their corresponding ground state. Suppose we now let $N$ go to infinity: why periodic geometries are favored energetically in this limit? In some very specific cases of pair interaction potential, and in some toy models of quantum nature in specific settings, the question can be solved: $[21,23,25,74,110,111,118,135,137]$. But in almost all cases relevant in practice, it is an unsolved theoretical question. Often, numerical tests are performed to obtain an answer. Therefore, in the absence of a proof that a model gives rise to a periodic structure for the most stable state of matter, it is useful to perform arguments (notably for the micro-to-macro limit) on non periodic structures. This is an additional motivation for the works overviewed in this section.

With a view to contributing to the clarification of the above issues, we introduced in [31], a setting for defining the energy of rather general microscopic structures. The following question is addressed:

What is the most general geometric property of an infinite set of nuclei (possibly accompanied by their cloud of electrons) that allows to define the average energy of the set?

Based on some very weak properties imposed to the set of nuclei (generalizing in some sense the assumptions (2.24) used above), we construct an algebra of functions, on which we set the variational problems that express the energy of the infinite set of particles.

Let us formally give two examples. The energy obtained in a pair-potential model reads

$$
I_{\text {nonperiodic }}^{\text {pair }}=\int_{\mathbb{R}^{3}} V(h) l(h) \mathrm{d} h
$$


where $l(h)$ is defined by

$$
l(h)=\lim _{R \rightarrow+\infty} \frac{1}{2 \#\left(B_{R} \cap\left\{\overline{x_{i}}\right\}\right)} \sum_{\overline{x_{i}} \in B_{R}} \sum_{\overline{x_{j}} \in B_{R} \backslash\left\{\overline{x_{i}}\right\}} \delta_{\overline{x_{i}}-\overline{x_{j}}} .
$$

The existence of (2.36) is the condition imposed on the set of sites, that generalizes the condition of periodicity, as can be easily seen. If the sites fill in the lattice $\mathbb{Z}^{3}$, we indeed have $l(h)=\frac{1}{2} \sum_{k \in \mathbb{Z}^{3} \backslash\{0\}} \delta_{k}$.

For a model quantum in nature, the conditions on the positions of nuclei are more stringent. The reason for this is that the interaction is an $N$-body interaction (with $N$ growing to infinity), and not simply a two-body interaction. Therefore, in the spirit of (2.36), correlations of positions at orders greater than 2 must be assumed to exist and be controlled. These conditions are made precise in [31]. We omit them here. Consider then a TFW model where the nuclei are smeared out (they consist not of Dirac masses, but of normalized nonnegative functions centered at points and compactly supported in a small region around these points). Then the energy of this infinite set of particles is defined to be ${ }^{3}$

$$
I_{\text {nonperiodic }}^{T F W}=\inf \left\{\mathcal{E}_{\text {nonperiodic }}^{T F W}(\rho),\langle\rho\rangle=\langle m\rangle,-\Delta \Phi=m-\rho\right\},
$$

where the energy functional is the averaged energy

$$
\mathcal{E}_{\text {nonperiodic }}^{T F W}(\rho)=C_{W}\left\langle|\nabla \sqrt{\rho}|^{2}\right\rangle+C_{T F}\left\langle\rho^{5 / 3}\right\rangle+\left\langle|\nabla \Phi|^{2}\right\rangle .
$$

In (2.38), $\langle f\rangle$ denotes the average value on large balls, i.e.

$$
\langle f\rangle=\lim _{R \longrightarrow+\infty} \frac{1}{\operatorname{meas}\left(B_{R}\right)} \int_{B_{R}} f(x) \mathrm{d} x,
$$

where $B_{R}$ denotes the ball of radius $R$ centered at the origin.

Although we did not compute the macroscopic limit of the models of this paragraph, we believe it is likely possible to do so, leading to a density of the type (2.13). We refer to the future work [34] for the details.

Stochastic lattices. A notion of stationary ergodic stochastic lattices has been introduced in [33] (see $[78,79]$ for a work in a similar setting). Such a notion generalizes the notion of periodic lattice in a broad sense, and in a direction somewhat different from that of the above paragraph. The space $(\Omega, \mathcal{F}, \mathbb{P})$ being a probability space, we consider a random variable $\ell$ valued in $\left(\mathbb{R}^{d}\right)^{\mathbb{Z}^{d}}$ :

$$
\begin{aligned}
\ell: \Omega & \longrightarrow\left(\mathbb{R}^{d}\right)^{\mathbb{Z}^{d}} \\
\omega & \longmapsto \ell(\omega)=\left\{\overline{x_{i}}, i \in \mathbb{Z}^{d}\right\} .
\end{aligned}
$$

This random variable formalizes our notion of stochastic lattice. Notice one of the advantage is that the lattice itself is the object manipulated. Therefore the numbering of the sites of the lattice becomes an irrelevant issue. This is in fact a major motivation for the introduction of this notion.

We also consider a group action $\left(\tau_{k}\right)_{k \in \mathbb{Z}^{d}}$ on $\Omega$, that preserves $\mathbb{P}$, and that is assumed ergodic (see $\left.[42,90]\right)$ :

$$
\left(\tau_{k} A=A, \forall k \in \mathbb{Z}^{d}\right) \quad \text { implies } \quad \mathbb{P}(A)=0 \quad \text { or } \quad 1 .
$$

\footnotetext{
${ }^{3}$ For the reader not familiar with Thomas-Fermi type models, it is not obvious to recognize in (2.37)-(2.38) the usual form (2.16)-(2.17) applied to smeared out nuclei. This is however true, and this can be seen with simple manipulations.
} 
The lattice $\ell$ is said a stationary ergodic stochastic lattice if

$$
\ell\left(\tau_{k} \omega\right)=\ell(\omega)-k, \quad \forall k \in \mathbb{Z}^{d} .
$$

Three simple examples of such lattices are:

(a) a periodic lattice

$$
\ell(\omega)=\left\{k, k \in \mathbb{Z}^{d}\right\},
$$

(b) a perturbation of a periodic lattice

$$
\ell(\omega)=\left\{k+X_{k}(\omega), \quad k \in \mathbb{Z}^{d}\right\}
$$

by independent identically distributed random variables $X_{k}(\omega)$ and

(c) the same perturbation but by stationary random variables

$$
X_{k}(\omega)=X_{0}\left(\tau_{k} \omega\right) .
$$

We do not really wish to give some physical content to the mathematical definition of lattices performed above. It is however tempting to see a setting including random perturbations of a periodic lattice as somehow related to the desire of modelling temperature effects. Combined with models rigorously defining the electronic structures at finite temperature (i.e. models generalizing the quantum models at zero temperature exposed here), such a setting would provide a ground for more satisfactory models. We will not pursue here in this direction, but it may be definitely interesting to do so.

In the simple case of a pair potential interaction, the techniques of Section 2.1.1, along with a repeated use of the ergodic theorem (see [32] for the details), allow to prove that for such a lattice deformed by a deformation $\varphi$, the energy per unit particle of the truncated lattice

$$
\frac{1}{\#(\ell(\omega) \cap \mathcal{D})} \sum_{\overline{x_{i}} \in \ell(\omega) \cap N^{1 / d} \mathcal{D}} \sum_{\overline{x_{j}} \in\left(\ell(\omega) \cap N^{1 / d} \mathcal{D}\right) \backslash\left\{\overline{x_{i}}\right\}} V\left(\frac{\varphi\left(\frac{\overline{x_{i}}}{N^{1 / d}}\right)-\varphi\left(\frac{\overline{x_{j}}}{N^{1 / d}}\right)}{1 / N^{1 / d}}\right)
$$

converges almost surely, in the limit $N \longrightarrow+\infty$, to

$$
\frac{1}{2 \mathbb{E}(\#(\ell(\omega) \cap Q))} \frac{1}{|\mathcal{D}|} \int_{\mathcal{D}} \mathbb{E}\left(\sum_{\overline{x_{i}} \in \ell(\omega) \cap Q} \sum_{\overline{x_{j}} \in \ell(\omega) \backslash\left\{\overline{x_{i}}\right\}} V\left(\nabla \varphi(x)\left(\overline{x_{i}}-\overline{x_{j}}\right)\right)\right) \mathrm{d} x,
$$

where $Q=\left[0,1\left[{ }^{d}\right.\right.$. The macroscopic density of mechanical energy therefore reads

$$
W(\nabla \varphi(x))=\mathbb{E}\left(\sum_{\overline{x_{i}} \in \ell(\omega) \cap Q} \sum_{\overline{x_{j}} \in \ell(\omega) \backslash\left\{\overline{x_{i}}\right\}} V\left(\nabla \varphi(x)\left(\overline{x_{i}}-\overline{x_{j}}\right)\right)\right)
$$

and is an obvious generalization of (2.11), again in the general form (2.13).

Again, various extensions of the above simple energy may be considered. In particular, the case of a microscopic scale modelled at the quantum level may be treated likewise. For brevity, we do not mention such extensions here.

The notion of stochastic lattice itself may even be further generalized, to avoid any relation to a reference lattice $\mathbb{Z}^{d}$ like in (2.40). All what is needed to define the microstructure is a notion of abstract measures $\mathcal{M}$ stationary with respect to an ergodic group action $\left(\tau_{x}\right)_{x \in \mathbb{R}^{d}}$ (notice the index of the group action is $x \in \mathbb{R}^{d}$ and no longer $\left.k \in \mathbb{Z}^{d}\right)$. Such a modelling gets rather technical and abstract. For this reason, let us only indicate, 
in a somewhat formal and vague manner, that a Thomas-Fermi-von Weizsäcker model (expressed here again in the case of smeared out nuclei for simplicity) will typically give rise to a thermodynamic limit of the type

$$
I_{\text {stat }}^{T F W}=\inf \left\{\mathcal{E}_{\text {stat }}^{T F W}(\rho), \rho(x, \omega)=R\left(\tau_{x} \omega\right), \sqrt{\rho} \in L^{1}\left(\Omega, H_{\text {loc }}^{1}\left(\mathbb{R}^{3}\right)\right), \mathbb{E}(R)=\mathbb{E}(\mathcal{M})\right\},
$$

where

$$
\mathcal{E}_{\text {stat }}^{T F W}(\rho)=C_{W} \mathbb{E}\left(|\nabla \sqrt{\rho}|^{2}\right)+C_{T F} \mathbb{E}\left(\rho^{5 / 3}\right)+\mathbb{E}\left(|\nabla \Phi|^{2}\right),
$$

i.e. a model analogous to (2.37)-(2.38), where the averages over large balls are replaced by expectation values. This is rather intuitive. The details may be read in [33]. The micro-to-macro limit will be investigated in [34].

\section{2. $\Gamma$-limit approaches}

In Section 2.1, we have worked in a setting that assumes the atoms of the solid are displaced by the macroscopic deformation $\varphi$. This is in particular equivalent to assuming that the deformation $\varphi$ is smooth at the atomic scale. However, as we already pointed out, this is a questionable hypothesis. It may in particular happen that a smooth macroscopic deformation arises from a non smooth microscopic one. More generally, the relation between the microscopic deformation and the macroscopic one is likely to be less simple than the identity we assumed. See for instance [73], for an example in which this hypothesis is not valid.

A means to explore this relation is to use $\Gamma$-convergence $[35,58]$ rather than the "pointwise" convergence we have used in [30]. In a nutshell, the approach consists in passing to the limit in the minimization problem defined by the energy rather than in the energy itself (we will recall the exact definition below). This allows to only consider the macroscopic deformation as the primary object, from which the microscopic deformation derivates. Such an approach was used in $[1,36-41,113]$. See also [136] for a related but somewhat different viewpoint.

Let us now briefly present this approach (details may be found in [1] and [39] for instance): we assume that the macroscopic reference state is a domain $\mathcal{D}$ of $\mathbb{R}^{d}$, and that the atoms inside this body occupy the periodic lattice $\varepsilon \mathbb{Z}^{3}$, where $\varepsilon=\frac{1}{N}$ is the interatomic distance. Assuming that a point $\frac{\overline{x_{k}}}{N}$ is transported to the point $\varphi\left(\frac{\overline{x_{k}}}{N}\right)$, the energy per particle $(2.7)$ then reads:

$$
F_{N}(\varphi)=\frac{1}{N^{3}} \sum_{\overline{x_{k}} \in \mathbb{Z}^{3} \cap N \mathcal{D}} \sum_{\overline{x_{i}} \in \mathbb{Z}^{3} \cap N \mathcal{D}} V\left(\frac{\varphi\left(\frac{\overline{x_{k}}}{N}\right)-\varphi\left(\frac{\overline{x_{i}}}{N}\right)}{\frac{1}{N}}\right) .
$$

Hence, following the method of [1], we may write:

$$
F_{N}(\varphi)=\sum_{\xi \in \mathbb{Z}^{3}} \sum_{\overline{x_{i}} \in \mathbb{Z}^{3} \cap N \mathcal{D}} \frac{1}{N^{3}} f^{\xi}\left(\frac{\varphi\left(\frac{\overline{x_{i}}+\xi}{N}\right)-\varphi\left(\frac{\overline{x_{i}}}{N}\right)}{\frac{|\xi|}{N}}\right),
$$

where we have set

$$
f^{\xi}(z)=V(|\xi| z)
$$

We have omitted some technical details related to the boundary terms (the second sum in (2.46) should not run over $\mathbb{Z}^{3} \cap N \mathcal{D}$ but only a part of it, depending on $\xi$ and $N$ ).

So far, $\varphi$ is only defined at the points of the lattice. We next identify it with its piecewise linear interpolation, and assume that it belongs to $L^{1}(\mathcal{D})$.

Before stating a result from [1], we recall for consistency the definition of $\Gamma$-convergence. We say that a sequence $F_{n}$ of functionals $\Gamma$-converges for the $L^{p}\left(\mathcal{D}, \mathbb{R}^{d}\right)$-topology to a functional $F$ if:

(1) (lower semicontinuity inequality) $\forall \varphi \in L^{p}\left(\mathcal{D}, \mathbb{R}^{d}\right)$, for all sequences $\varphi_{n}$ converging to $\varphi$ in $L^{p}\left(\mathcal{D}, \mathbb{R}^{d}\right)$, we have $F(\varphi) \leq \liminf F_{n}\left(\varphi_{n}\right)$

(2) (existence of a recovery sequence) $\forall \varphi \in L^{p}\left(\mathcal{D}, \mathbb{R}^{d}\right)$, there exists a sequence $\varphi_{n}$ converging to $\varphi$ in $L^{p}\left(\mathcal{D}, \mathbb{R}^{d}\right)$ such that $F(\varphi)=\lim F_{n}\left(\varphi_{n}\right)$. 
This definition is to be related to the problem of the convergence of the corresponding minimization problems. Consider

$$
I_{n}=\inf \left\{F_{n}(\psi), \quad \psi \in V\right\},
$$

where $V$ is some subspace of $L^{p}(\mathcal{D})$. Then, the fact that $F_{n} \Gamma$-converges to $F$ in particular implies that if a sequence $\varphi_{n}$ of minimizers of $I_{n}$ converges to some $\varphi$, then

(1) $\varphi$ is a solution of

(2) we have

$$
I=\inf \{F(\psi), \quad \psi \in V\}, \quad \text { and }
$$

$$
\lim _{n \rightarrow \infty} I_{n}=I .
$$

Of course, the convergence of the sequence of minimizers $\varphi_{n}$ (or more generally its compactness) is not true unconditionally. Some coercivity assumptions on the functionals $F_{n}$ are needed to obtain such a result (see [58]). This however indicates that in favorable cases, $I_{n}$ converges to $I$, and the minimizers of $I_{n}$ are convenient approximations of the minimizer(s) of $I$.

Theorem 2.1 (Thm. 3.1 of [1]). Assume that $f^{\xi} \geq 0$ satisfies the following assumptions (here, $\left(e_{1}, e_{2}, e_{3}\right)$ is the canonical basis of $\left.\mathbb{R}^{3}\right)$ : there exists $p>1$ such that

$$
\begin{aligned}
& \exists c_{1}>0, \quad \forall z \in \mathbb{R}^{d}, \quad \forall i \in\{1,2,3\}, \quad f^{e_{i}}(z) \geq c_{1}\left(|z|^{p}-1\right), \\
& \exists C_{N}^{\xi}, \quad \forall z \in \mathbb{R}^{d}, \quad f^{\xi}(z) \leq C_{N}^{\xi}\left(|z|^{p}+1\right), \\
& \sum_{\xi \in \mathbb{Z}^{d}} C_{N}^{\xi}<+\infty .
\end{aligned}
$$

Then, there exists a sequence $N_{k}$ going to infinity and a quasiconvex ${ }^{4}$ function $f: \mathbb{R}^{3 \times 3} \rightarrow[0,+\infty]$ such that

$$
c\left(|M|^{p}-1\right) \leq f(M) \leq C\left(|M|^{p}+1\right),
$$

for some constants $0<c<C$, and such that $F_{N_{k}} \Gamma$-converges in the $L^{p}\left(\mathcal{D}, \mathbb{R}^{d}\right)$-topology to the functional $F$ defined by

$$
F(\varphi)= \begin{cases}\int_{\mathcal{D}} f(\nabla \varphi) \mathrm{d} x & \text { if } \varphi \in W^{1, p}\left(\mathcal{D}, \mathbb{R}^{3}\right) \\ +\infty & \text { otherwise. }\end{cases}
$$

Moreover, $f$ is defined by (here, $Q=\left[-\frac{1}{2}, \frac{1}{2}\right]^{3}$ is the unit cube of $\mathbb{R}^{3}$ )

$$
f(M)=\lim _{R \rightarrow+\infty} \frac{1}{R^{3}} \min \left\{\sum_{\xi \in \mathbb{Z}^{d}} \sum_{\beta \in \mathbb{Z}^{3}, \xi+\beta \in R Q} f^{\xi}\left(\frac{\psi(\beta+\xi)-\psi(\beta)}{|\xi|}\right), \quad \psi \in \mathcal{A}_{M}(R Q)\right\},
$$

with $\mathcal{A}_{M}(R Q)=\left\{\psi \in C^{0}(R Q), \quad \psi(\alpha)=M \alpha \quad\right.$ if $\left.\quad(\alpha+Q) \cap(R Q)^{c} \neq \emptyset\right\}$.

Theorem 3.1 of [1] is in fact more general than the simple form stated above. We have simplified it here to avoid unnecessary technicalities, and to fit into the present context of energies of the type (2.1) defined in Section 2.

Some remarks are in order. First, the functional $F$ in Theorem 2.1 is not explicit, except in very specific cases. The setting of nearest-neighbor interactions is such a case. Then, $f^{\xi}$ is defined by

$$
f^{\xi}(z)= \begin{cases}V(z) & \text { if }|\xi|=1 \\ 0 & \text { otherwise }\end{cases}
$$

\footnotetext{
${ }^{4}$ This notion is recalled in Definition 3.1 below.
} 
This definition of $f^{\xi}$ implies that (2.48) to (2.50) are satisfied. In addition, in dimension one, we then have (see [41]):

$$
f(z)=V^{* *}(z),
$$

where $V^{* *}$ is the convex enveloppe of $V$. Note that in the pointwise approach of Section 2.1, the limit is

$$
f(z)=V(z) .
$$

The energy densities obtained respectively read

$$
W\left(\varphi^{\prime}(x)\right)=V^{* *}\left(\varphi^{\prime}(x)\right),
$$

and

$$
W\left(\varphi^{\prime}(x)\right)=V\left(\varphi^{\prime}(x)\right)
$$

They are thus different, except in the special case of a convex interaction potential $V$. Taking the example of the Lennard-Jones potential, one thus gets:

$$
V(z)=\frac{1}{z^{12}}-\frac{2}{z^{6}}, \quad \text { and } \quad V^{* *}(z)= \begin{cases}V(z) & \text { if } z<1 \\ -1 & \text { otherwise }\end{cases}
$$

Note however that the quasiconvex envelop of (2.53) and (2.54) are the same, so that, as we will see in Section 3.1 below, the corresponding minimization problems enjoy the same properties.

Formula (2.53) can be generalized to higher dimensions [39]. In [41], other formulas are derived in the case of next to nearest neighbor interaction. It seems however, as pointed out in [39], that (2.52) cannot be made explicit even in dimension one in the case of interactions of larger range.

A second comment concerns hypotheses (2.48) to (2.50), that are seldom satisfied by the definition (2.47) of $f^{\xi}$. For instance, imposing (2.48) implies some growth at infinity for the interaction potential, which has to go to infinity at infinity. However, in the case of finite range interaction, for a suitable $V$, (2.48) to (2.50) may hold. Note also that in dimension one, a result similar to Theorem 2.1 has been proved in [39] for potentials which may go to zero at infinity, therefore by passing (2.48).

\section{The Multiscale MOdels}

Section 2 provides hyperelastic functionals of the type (1.7), where the energy density $W$ may or may not be explicit, but accounts for the underlying atomic structure.

Using (1.7), a way to define the deformation $\varphi$ of a solid subjected to a force $f$ and with boundary conditions written in an abstract way $\varphi \in \mathcal{A}$, is to solve the following minimization problem (1.5), which we recall here:

$$
\inf _{\varphi \in \mathcal{A}} \int_{\mathcal{D}} W(\nabla \varphi(x)) \mathrm{d} x-\int_{\mathcal{D}} f \varphi .
$$

The set $\mathcal{A}$ may be for instance defined as follows:

$$
\mathcal{A}=\left\{\varphi \in W^{1, p}\left(\mathcal{D}, \mathbb{R}^{3}\right), \quad \forall x \in \partial \mathcal{D}, \quad \varphi(x)=M x\right\},
$$

where $M \in \mathbb{R}^{3 \times 3}$ is a given matrix, and $p>1$ is a real number related to $W$ (see Thm. 3.1 below). When the functional $W$ is supposed to be computed according to some microscopic model, for instance with (2.11), (2.27), or more generally (2.13), this formula is in some sense a multiscale model. However, if the computational resources were not limited, it is usually considered that the minimization problem to consider would be:

$$
\inf _{\varphi \in \mathcal{A}_{N}} \frac{1}{N^{d}}\left(\sum_{\overline{x_{i}} \neq \overline{x_{j}} \in \frac{1}{N} \mathbb{Z}^{d} \cap \mathcal{D}} V\left(\frac{\varphi\left(\overline{x_{i}}\right)-\varphi\left(\overline{x_{j}}\right)}{\frac{1}{N}}\right)-\sum_{\overline{x_{i}} \in \frac{1}{N} \mathbb{Z}^{d} \cap \mathcal{D}} f\left(\overline{x_{i}}\right) \varphi\left(\overline{x_{i}}\right)\right) .
$$




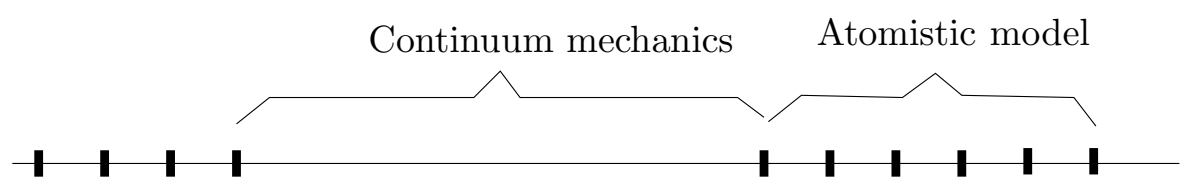

Figure 1. Partition of $\mathcal{D}$ (in dimension one) into a regular zone $\mathcal{D}_{\text {reg }}$ where the continuum mechanics model is used, and a singular zone $\mathcal{D}_{\text {sing }}$ where the atomistic model is used.

The fact that (3.3) is the reference model is sometimes a controversy issue, that we shall not consider here. In any event, the energy (3.1) is a good approximation of (3.3) if the deformation $\varphi$ is smooth. Apart from the numerous situations in which $\varphi$ is indeed smooth, and thus (3.1) is a suitable model, the question of dealing with possible singularities of $\varphi$ may be asked. Fortunately, in most cases, these singularities are localized in small regions of $\mathcal{D}$, and the sum in (3.3) if restricted to these regions is not that large. It is thus a natural strategy to use (3.1) in the region where $\varphi$ is smooth, and resort to (3.3) in the (hopefully small) region where $\varphi$ have singularities. This leads to a minimization problem involving the energy (1.8), where $\mathcal{D}_{\text {reg }}$ is the subdomain of $\mathcal{D}$ where $\varphi$ is supposed to be smooth, and $\mathcal{D}_{\text {sing }}$ its complementary set (see Fig. 1):

$$
\inf _{\varphi \in \tilde{\mathcal{A}}_{N}}\left\{\int_{\mathcal{D}_{\text {reg }}} W(\nabla \varphi(x)) \mathrm{d} x-\int_{\mathcal{D}_{\text {reg }}} f \varphi+\frac{1}{N^{d}}\left(\sum_{\overline{x_{i}} \neq \overline{x_{j}} \in \varepsilon \mathbb{Z}^{d} \cap \mathcal{D}_{\text {sing }}} V\left(\frac{\varphi\left(\overline{x_{i}}\right)-\varphi\left(\overline{x_{j}}\right)}{\varepsilon}\right)-\sum_{\overline{x_{i}} \in \varepsilon \mathbb{Z}^{d} \cap \mathcal{D}_{\text {sing }}} f\left(\overline{x_{i}}\right) \varphi\left(\overline{x_{i}}\right)\right)\right\} .
$$

Note that this model raises the problem of defining $\mathcal{D}_{\text {reg }}$ in an adaptive way.

The following subsections are dedicated to the mathematical and numerical study of problems (3.1) and (3.4). Section 3.1 gives an account of theoretical results on problem (3.1). On the other hand, Section 3.2 presents a study of problems (3.3) and (3.4), in a particularly simple case. Section 3.3 is a discussion about alternative ways for computing the equilibrium deformation, that do not consider global minimization problems, as we did above in Section 2 and thus in formula (3.4).

\subsection{Mathematical study of the continuum problem}

This subsection deals with problem (3.1). It may be seen as a preliminary step before studying the coupled problem (3.4), since (3.1) is in some sense a special case of (3.4) with $\mathcal{D}_{\text {sing }}=\emptyset$. A very nice review on this kind of mathematical problems may be found in [10].

In order to study (3.1), we first need to introduce the notion of quasiconvexity $[8,108]$ :

Definition 3.1. Let $\mathcal{D}$ be a domain of $\mathbb{R}^{d}$, with $d \in \mathbb{N}$. Let $W: \mathbb{R}^{d \times d} \longrightarrow \mathbb{R}$ be a continuous function. We say that $W$ is quasiconvex if

$$
\forall M \in \mathbb{R}^{d \times d}, \quad \forall \xi \in C_{0}^{\infty}\left(\mathcal{D}, \mathbb{R}^{d}\right), \quad \int_{\mathcal{D}} W(M x+\nabla \xi(x)) \mathrm{d} x \geq|\mathcal{D}| W(M)
$$

Although this definition seems to depend on the domain $\mathcal{D}$, it does not. This is proved in [108] and [13]. Actually, quasiconvexity may be shown, under some fairly general hypotheses, to be equivalent to the lower semi-continuity of the functional $\varphi \mapsto \int W(\nabla \varphi)[8,108]$. Thus, the minimization problems of the form $(3.1)$ have minimizers.

The main difficulty is that quasiconvexity is a nonlocal condition [10], in general difficult to check. It has even been shown in [91] that in dimension $d \geq 3$, quasiconvexity cannot be rewritten as a local property. Therefore other notions related to quasiconvexity (namely polyconvexity, rank-one convexity) have been derived. They give necessary or sufficient conditions for quasiconvexity, which are easier to manipulate. See for instance $[8,9,57,109,115,129]$ for more details. 
The fundamental result in this setting is:

Theorem 3.1. [13] Assume that $W$ is quasiconvex and bounded from below and that $f \in L^{1}(\mathcal{D})$. Assume in addition that $W$ satisfies the growth condition:

$$
\exists p>1, \quad \exists C_{2} \geq C_{1}>0, \quad \text { such that } \forall M \in \mathbb{R}^{3 \times 3}, \quad C_{1}\left(|M|^{p}-1\right) \leq W(M) \leq C_{2}\left(|M|^{p}+1\right),
$$

and that $\mathcal{A}$ is defined by (3.2) with the same $p$ as in (3.5). Then, problem (3.1) has a minimizer $\varphi \in W^{1, p}(\mathcal{D})$.

Note that this result also holds in dimensions different than 3. However, this result does not apply to most physically relevant cases. In particular, a natural assumption is that matter cannot interpenetrate itself, which mathematically translates into the property $W(M) \longrightarrow+\infty$ as $\operatorname{det}(M) \longrightarrow 0^{+}$. This is clearly in contradiction with (3.5). In the same spirit, the expression (2.11) implies invariances which prevent $W$ to be quasiconvex (we refer to [70] for the proof, and to [54,69] for related results), unless it is a function of the determinant of $\nabla \varphi(x)$.

As an example of a situation outside the setting of Theorem 3.1, let us consider the so-called one-well problem: we assume that $f=0$ (although what follows carries through the case of nonzero forces), and that $W$ is given by

$$
W(F)=d(F, S O(3)):=\inf _{R \in S O(3)}\|F-R\|^{2},
$$

where $\|M\|^{2}=\operatorname{Trace}\left({ }^{t} M M\right)$, and where $S O(3)$ is the group of rotations in $\mathbb{R}^{3}$. Obviously, $W$ is nonnegative and vanishes only on $S O(3)$. This functional satisfies the frame invariance. According to [82] (see also [109]), we have (recall that $f=0$ ):

- if $\varphi$ is a minimizer of (3.1), with $\mathcal{A}$ defined by (3.2), then $\varphi(x)=M x+b$ almost everywhere in $\mathcal{D}$, for some $b \in \mathbb{R}^{3}$;

- any minimizing sequence $\left(\varphi_{j}\right)_{j \in \mathbb{N}}$ of $(3.1)$ satisfies $\nabla \varphi_{j} \longrightarrow M$ in measure.

The second point is far from trivial. The gradient of a minimizing sequence is easily shown to converge almost everywhere to a matrix of the form $R M$, where $R \in S O(3)$ may depend on $x \in \mathcal{D}$. The point is to show that in fact this rotation $R$ is constant. The result is closely related to fundamental results contained in $[72,80,81,119]$ and called rigidity lemmas.

More involved is the case when $W$ in (3.1) has two minima. More precisely, we assume that $W \geq 0$, and that there exists two matrices $A \neq B$ such that

$$
W(M)=0 \Longleftrightarrow(M \in A S O(3) \text { or } \quad M \in B S O(3)) .
$$

We then have the following property [11]: if $\varphi \in W^{1, \infty}(\mathcal{D})$ is such that for all $x \in \mathcal{D}, \nabla \varphi(x) \in\{A, B\}$, then:

- if $\operatorname{rank}(A-B) \geq 2$, then $\nabla \varphi=A$ almost everywhere or $\nabla \varphi=B$ almost everywhere;

- if $A-B=a \otimes n$, where $a \in \mathbb{R}^{3}$ and $n \in \mathbb{R}^{3}$, then there exists a Lipschitz function $h$ such that $h^{\prime} \in\{0,1\}$ and

for some $b \in \mathbb{R}^{3}$.

$$
\varphi(x)=A x+a h(x \cdot n)+b,
$$

Hence, in the first case, i.e. if $A$ and $B$ are not rank-one connected, then $\nabla \varphi$ must be constant, and the behavior is the same as in the one-well problem. On the contrary, in the second case, i.e. if $A$ and $B$ are rank-one connected, minimizing sequences may behave differently. To see this, assume in addition to (3.7) that the boundary condition $M$ satisfies

$$
M=\lambda A+(1-\lambda) B, \quad \text { for some } \quad \lambda \in(0,1) .
$$

Then it is possible to construct a minimizing sequence of (3.1) as follows. We introduce

$$
h(t)=\left\{\begin{array}{lll}
-(1-\lambda) t & \text { if } & t \in[0, \lambda), \\
\lambda(t-1) & \text { if } & t \in[\lambda, 1]
\end{array}\right.
$$




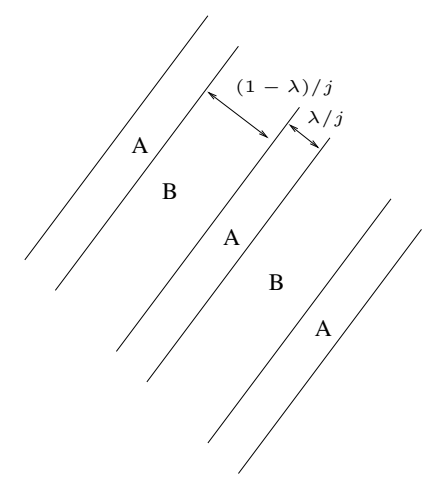

FiguRE 2. The laminate structure described by (3.8).

which is Lipschitz continuous. We then set

$$
\varphi_{j}(x)=\lambda A x+(1-\lambda) B x+\frac{1}{j} a h(j x \cdot n) .
$$

We then have $\nabla \varphi_{j} \in\{A, B\}$ almost everywhere in $\mathcal{D}$. Hence, up to boundary conditions, $\left(\varphi_{j}\right)_{j \in \mathbb{N}}$ is a minimizing sequence of (3.1). In order to satisfy boundary conditions, consider a cut-off function $\chi \in C^{\infty}([0,+\infty))$ such that $0 \leq \chi \leq 1, \chi=0$ in $[0,1 / 2]$ and $\chi=1$ in $[1, \infty)$, and define

$$
\tilde{\varphi}_{j}(x)=\chi(j \operatorname{dist}(x, \partial \mathcal{D})) \varphi_{j}(x) .
$$

This is a minimizing sequence of (3.1) (shown in Fig. 2), which satisfies $\nabla \tilde{\varphi}_{j}=\nabla \varphi_{j}$ except in a strip of thickness $1 / j$ around $\partial \mathcal{D}$ (not displayed in Fig. 2).

This example demonstrates that if the boundary condition $M$ and the matrices $A$ and $B$ satisfy some appropriate compatibility conditions, then minimizing sequences of (3.1) may asymptotically develop arbitrarily fine oscillations. In order to describe such oscillations, gradient Young measures $[17,109,114,139]$ are introduced. First we state

Theorem 3.2 (definition of Young measures [139]). Let $u_{j}: \mathcal{D} \longrightarrow \mathbb{R}^{3 \times 3}$ be a sequence of measurable functions such that

$$
\lim _{M \rightarrow+\infty} \sup _{j \in \mathbb{N}}\left|\left\{\left|u_{j}\right| \geq M\right\}\right|=0 .
$$

Then there exists a subsequence $u_{j_{k}}$ and a weak-* measurable map $\nu$ from $\mathcal{D}$ to the set $\mathcal{M}\left(\mathbb{R}^{3 \times 3}\right)$ of measures on $\mathbb{R}^{3 \times 3}$ such that

$$
\begin{array}{r}
\nu_{x} \geq 0, \quad\left\|\nu_{x}\right\|_{\mathcal{M}\left(\mathbb{R}^{3 \times 3}\right)}=\int_{\mathbb{R}^{3 \times 3}} \mathrm{~d} \nu_{x}=1 \quad \text { for almost every } \quad x \in \mathcal{D}, \\
\forall f \in C^{0}\left(\mathbb{R}^{d}\right), \quad f\left(u_{j_{k}}\right) \stackrel{*}{\longrightarrow} \bar{f} \quad \text { in } \quad L^{\infty}(\mathcal{D}), \quad \text { where } \bar{f}(x)=\left\langle\nu_{x}, f\right\rangle=\int_{\mathbb{R}^{3 \times 3}} f(y) \mathrm{d} \nu_{x}(y) .
\end{array}
$$

The measure $\nu_{x}$ is called a Young measure generated by the sequence $u_{j}$.

In the above example, the sequence $\nabla \varphi_{j}$ is easily seen to generate the Young measure

$$
\nu_{x}=\lambda \delta_{A}+(1-\lambda) \delta_{B} .
$$

Loosely speaking, the Young measure $\nu_{x_{0}}$ gives the probability of finding a given matrix in the sequence $u_{j_{k}}(x)$ for $x$ in a small neighborhood $B_{r}\left(x_{0}\right)$ in the limits $k \rightarrow \infty$ and $r \rightarrow 0$. 
Consider now the set $\mathcal{B}$ of Young measures generated by sequences of gradients of $W^{1, p}$ functions: they are called $W^{1, p}$ gradient Young measures. It is possible to give a characterization of $\mathcal{B}$ in relation with quasiconvexity (see [83]), but we will not do so. However, a nice interpretation of problem (3.1) is given by the following result, proved in [84]: let

$$
\mathcal{B}=\left\{\nu: \mathcal{D} \longrightarrow \mathcal{M}\left(\mathbb{R}^{3 \times 3}\right), \quad \nu \quad \text { is a gradient Young measure, } \quad \exists \varphi \in \mathcal{A}, \quad \forall x \in \mathcal{D}, \quad\left\langle\nu_{x}, \mathrm{id}\right\rangle=\nabla \varphi(x)\right\} .
$$

Then problem (3.1) may be recast into the setting of gradient Young measures as follows (we assume here that $W$ satisfies (3.5)):

$$
\inf \left\{\int_{\mathcal{D}} W(\nabla \varphi(x)) \mathrm{d} x, \quad \varphi \in \mathcal{A}\right\}=\min \left\{\int_{\mathcal{D}}\left\langle\nu_{x}, W\right\rangle \mathrm{d} x, \quad \nu \in \mathcal{B}\right\}
$$

Indeed, by extending the variational space $\mathcal{A}$ to the corresponding gradient Young measure space $\mathcal{B}$, one obtains a minimization problem which has at least one minimizer. In addition, the minimizers of the right-hand side of (3.9) are gradient Young measures generated by gradients of minimizing sequences of the minimization problem of the left-hand side. Of course, the above construction is consistent: if the first has a minimizer $\varphi$, then a minimizer for the right-hand side of (3.9) is $\nu_{x}=\delta_{\nabla \varphi(x)}$ almost everywhere.

This relaxation procedure is well-suited for general non quasiconvex problems, and especially for microstructures. However, once the variational space has been enlarged in this way, it remains to compute numerically the corresponding minimizer. The practical difficulty here is that both the domain $\mathcal{D}$ and the space $\mathbb{R}^{3 \times 3}$ of matrices need to be discretized. The computational load is thus significant, not to say overwhelming, in actual situations. We refer to [47] for a numerical analysis of this kind of problem.

The above results concerning one-well and double-well problems have (to some extent) their counterpart for the three-well and four-well problem. There is a huge amount of literature on these subjects. We refer for instance to $[8,11,12,18,19,57,61,89,109,115,128]$ and the references therein, without being exhaustive. Note that in dimension two, the two-well problem is completely analyzed in [130].

Despite many theoretical results, a question remains. As the above analysis shows for the two-well problem, infinitely fine microstructures may appear. In experiments, they of course have finite thickness. This apparent contradiction owes to the fact that the natural length scale (i.e. the atomic distance) is absent in (3.1). Actually, for instance for martensitic transitions, one may observe layers having thickness of a few atoms. A typical explanation for this absence of any length scale in (3.1) is to remark that we have sent the interatomic distance to zero in the limiting processes of Section 2. Of course, restoring the discrete energy of (3.3) would be a means to reinstall a characteristic length in the problem, but, as pointed out above, this is computationally very costly. Hence, the coupled problem (3.4) may be seen as a pragmatic compromise.

\subsection{Analysis of a coupled problem in dimension 1}

This subsection is devoted to the study of the coupled problem (3.4). For clarity, we perform the analysis in a one-dimensional setting, as in $[27,28]$. The generalization to higher dimensions is not clear for now, but as we will see below, serious difficulties arise even in this simple case. We thus assume that $\mathcal{D}=(0,1)$, and first consider the discrete problem (3.3). We use here a simpler notation for the equilibrium configuration of atoms:

$$
\overline{x_{i}}=\frac{i}{N}, \quad i \in\{1,2, \ldots, N\} .
$$

We assume that $f=0$, that $V$ is the Lennard-Jones potential

$$
V(r)=\frac{1}{r^{12}}-\frac{2}{r^{6}}
$$


and we only account for nearest neighbor interactions. The discrete problem (3.3) therefore takes the simpler following form:

$$
\inf _{\varphi \in \mathcal{A}_{N}} \frac{1}{N}\left(\sum_{i=0}^{N-1} V\left(\frac{\varphi\left(\frac{i+1}{N}\right)-\varphi\left(\frac{i}{N}\right)}{\frac{1}{N}}\right)\right) .
$$

The the minimization set $\mathcal{A}_{N}$ is defined by

$$
\mathcal{A}_{N}=\left\{\varphi:\left\{0, \frac{1}{N}, \ldots, \frac{N-1}{N}, 1\right\} \rightarrow \mathbb{R}, \quad \varphi(0)=0, \quad \varphi(1)=a, \quad \varphi \text { non decreasing }\right\},
$$

where $a>0$ is a given length. The monotonicity condition in (3.11) accounts for the fact that only injective deformations $\varphi$ are admissible (this formulation is a consequence of the dimension).

As we will see, even in this simple setting, non trivial difficulties arise, which we think are prototypical of more general situations.

If $a$ is sufficiently close to 1 , the solution of (3.3) is expected to be the affine function:

$$
\varphi\left(\frac{i}{N}\right)=a \frac{i}{N}
$$

However, as proved in [27], if $N$ is large enough, we have

- If $a \leq 1$, then (3.3) has a unique minimizer defined by $\varphi\left(\frac{i}{N}\right)=i \frac{a}{N}$.

- If $a>1$, then (3.3) has infinitely many minimizers that we now describe (see Fig. 3). There exists a unique pair of real numbers $\left(s, s_{f}\right) \in \mathbb{R}^{2}$ such that

$$
1 \leq s \leq 1+\frac{1}{N}, \quad V^{\prime}(s)=V^{\prime}\left(s_{f}\right) \quad \text { and } \quad\left(1-\frac{1}{N}\right) s+\frac{1}{N} s_{f}=a,
$$

and for any minimizer of (3.3), there exists one and only one index $i_{\mu}$ such that

$$
\forall i \neq i_{\mu}, \frac{\varphi\left(\frac{i+1}{N}\right)-\varphi\left(\frac{i}{N}\right)}{\frac{1}{N}}=s \quad \text { and } \quad \frac{\varphi\left(\frac{i_{\mu}+1}{N}\right)-\varphi\left(\frac{i_{\mu}}{N}\right)}{\frac{1}{N}}=s_{f} .
$$

The physical interpretation of (3.13)-(3.14) is the following: if the material under consideration is in extension, which corresponds to $a>1$, then the material breaks. This is the meaning of (3.14): $s$ converges to 1 as $N$ tends to infinity (and recall that $N$ is large), and $s_{f} \approx N a$. Such results are already known in the literature (see $[40,67,136])$.

Remark 3.1. The fact that for any $a>1$ the material breaks may seem rather unphysical at first sight. However, it seems that for very low temperature, metals may behave in such a way. It is therefore an indication that temperature effects are very important in this kind of problems.

We now turn to the coupled problem (3.4). Assuming that $\mathcal{D}=\mathcal{D}_{\text {reg }} \cup \mathcal{D}_{\text {sing }}$ is a nontrivial partition of $\mathcal{D}$, we assume for simplicity that

$$
\mathcal{D}_{\text {sing }}=(0, b], \quad \text { with } \quad b<1 .
$$

The set $\mathcal{A}$ reads

$$
\mathcal{A}=\left\{\begin{array}{c}
\varphi ; \varphi_{\mid \mathcal{D}_{\text {reg }}} \in W^{1,1}\left(\mathcal{D}_{\text {reg }}\right), \varphi_{\mid \mathcal{D}_{\text {sing }}} \text { is the discrete set of } \\
\text { variables } \varphi\left(\frac{i}{N}\right), \varphi(0)=0, \varphi(1)=a, \\
\varphi \text { is increasing on } \mathcal{D}
\end{array}\right\} .
$$




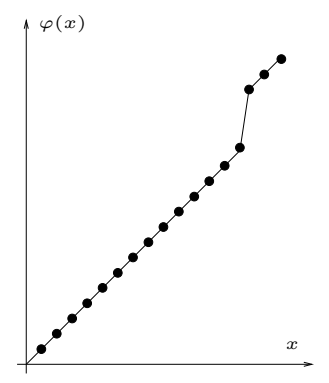

Figure 3. The deformed state described by (3.14).

We assume that $\mathcal{D}_{\text {reg }}$ and $\mathcal{D}_{\text {sing }}$ have been chosen such that we expect that singularities of the deformation are

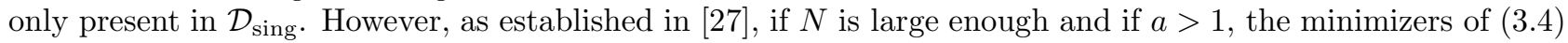
are of the following form:

$$
\begin{aligned}
\forall \frac{i}{N} \in \mathcal{D}_{\text {sing }}, \quad \varphi\left(\frac{i}{N}\right) & =\frac{i}{N} \\
\forall x \in \mathcal{D}_{\text {reg }}, \quad \varphi(x) & =x+\sum_{i \in \mathbb{I}} \tilde{v}_{i} H\left(x-x_{i}\right), \quad \forall i, x_{i} \in \mathcal{D}_{\text {reg }}, \tilde{v}_{i}>0,
\end{aligned}
$$

with $\mathbb{I} \subset \mathbb{N}$ and $\sum_{i \in \mathbb{I}} \tilde{v}_{i}=a-L$. Here, $H$ denotes the Heaviside function: $H(t)=0$ if $t<0, H(t)=1$ if $t>0$.

This indicates that, as in the fully discrete model (3.3) studied above, the material breaks if $a>1$. However, the fracture systematically occurs in the regular zone $\mathcal{D}_{\text {reg, }}$, that is in the zone where $\varphi$ is expected to be smooth.

Although the complete analysis of problem (3.4) is rather technical, a simple argument on the energy shows that if a fracture occurs, it necessarily does in the regular zone $\mathcal{D}_{\text {reg. }}$. Indeed, assume that $a>1$, and define the configuration $\varphi_{1}$ which has a fracture in $\mathcal{D}_{\text {sing }}$ and $\varphi_{2}$ which has a fracture in $\mathcal{D}_{\text {reg }}$, namely (here $i_{0} \in \mathbb{N}$ is such that $\frac{i_{0}}{N} \in \mathcal{D}_{\text {sing }}$ and $x_{0} \in \mathcal{D}_{\text {reg }}$ ):

$$
\begin{array}{cl}
\forall i \leq i_{0}, & \varphi_{1}\left(\frac{i}{N}\right)=\frac{i}{N}, \\
\forall i_{0}<i \leq N b, & \varphi_{1}\left(\frac{i}{N}\right)=\frac{i}{N}+a-1, \\
\forall x \in \mathcal{D}_{\text {reg }}, & \varphi_{1}(x)=x+a-1,
\end{array}
$$

and

$$
\begin{aligned}
\forall i \leq N b, & \varphi_{2}\left(\frac{i}{N}\right)=\frac{i}{N} \\
\forall x \in \mathcal{D}_{\text {reg }}, & \varphi_{2}(x)=x+(a-1) H\left(x-x_{0}\right) .
\end{aligned}
$$

Such a deformation $\varphi$ is displayed in Figure 4 .

Computing the corresponding coupled energy (3.4), denoted by $E_{i}$ for $E\left(\varphi_{i}\right)$ yields:

$$
E_{1}=\left(1-\frac{1}{N}\right) V(1)+V(1+N(a-1))=-1+\frac{1}{N}+O\left(\frac{1}{N^{7}}\right), \quad E_{2}=V(1)=-1 .
$$

Hence, the configuration $\varphi_{1}$, for which the fracture lies in $\mathcal{D}_{\text {sing }}$, has an energy higher than the configuration $\varphi_{2}$, for which the fracture is in $\mathcal{D}_{\text {reg }}$. 


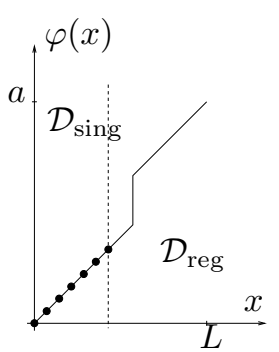

FIGURE 4. The deformed state described by (3.18)-(3.19).

Note however that the difference of energy is of order $\frac{1}{N}$, which is tiny (recall that $N \sim 10^{23}$ ). In addition, the above argument assumes an exact evaluation of the energies. In practice, the energy of the continuous model is evaluated using a discretization method (say FEM for simplicity), which has a finite mesh size $h$. Hence, $E(\varphi)=\int V\left(\varphi^{\prime}\right)$ is approximated by

$$
E^{h}(\varphi)=\frac{1}{N_{h}} \sum_{j=1}^{N_{h}} V\left(\varphi^{\prime}\left(x_{j}\right)\right) .
$$

Inserting (3.19) into this energy yields

$$
E_{2}^{h}=E^{h}\left(\varphi_{2}\right)=-1+\frac{1}{N_{h}}+o\left(\frac{1}{N_{h}}\right), \quad \text { while } \quad E_{1}^{h}=E^{h}\left(\varphi_{1}\right)=E_{1}
$$

given by (3.20). As the number $N_{h}$ is in general much smaller than the number of atoms $N$, this compensates for the difference in energy. It follows that, although the theory establishes the fracture always occur in the continuous zone $\mathcal{D}_{\text {reg }}$, the practice, which implements a discretized approximation of the continuous energy, shows a different behavior. A numerical artifact somehow saves the situation.

This discussion certainly demonstrates that properly setting problem (3.4) is uneasy. Although the above study is only carried out in a one-dimensional setting, similar difficulties may be expected in more general situations. The simple mathematical observation performed above, along with the absence of a complete mathematical analysis of the situation (in particular regarding the systematic study for choosing $\mathcal{D}_{\text {reg }}$ ) indicate that all results obtained with this kind of coupled method must be taken with great care. The definite practical success of numerical approaches involving hybrid problems of the type (3.4) should motivate further mathematical efforts.

With this objective in mind, let us conclude the present section by somehow modulating the above mathematical observation. The above results heavily rely on the fact that the interaction potential vanishes at infinity. However, in the case when the potential is convex (with for instance nearest neighbor interactions, and with suitable coercivity assumptions), a simple analysis, still in dimension one [28], proves that problem (3.4) has a unique solution, and that this solution converges, as $N$ goes to infinity, to the solution of (3.1), which is also unique. In addition, the solution of (3.4) converges to that of (3.3) as $N$ goes to infinity. This indicates that in this case, contrary to the Lennard-Jones case, the coupled model is a good approximation of the fully discrete model.

\subsection{Local rather than global minimization}

Let us now return to the fully discrete problem (3.10)-(3.11) with Lennard-Jones potential in dimension one. The results from [27] we quoted in Section 3.2 show that the predictions of this model do not agree with experiment. Bars under arbitrarily small stretching should not break (see however Rem. 3.1 above).

We have only considered here the discrete model, but the corresponding macroscopic limit exhibits the same behavior. In the definition of the problem, we have two ingredients: the first one is the energy functional, the 
second one is the fact that we minimize the energy. Actually, both ingredients are questionable, and they are related to one another.

The problems considered above are global minimizations of energies at zero temperature. As far as the electronic structure of the material is concerned, this seems like a fair assumption, considering the extremely rapid relaxation time of the electrons. In contrast, this is a more questionable assumption for the nuclei. In addition, models accounting for finite temperature effects could be alternatively considered: they would consist of the minimization of the free energy, rather than the energy, of the nuclei (or in the classical setting, of the atoms). To some extent, our work [33], outlined in Section 2.1 above, is a first step in this direction.

A related issue is to go beyond global minimization.

Indeed, the example of Section 3.2 indicates that the physically relevant state, defined by (3.12), is a critical point of the energy which is not the minimizer. In fact, the hessian of the energy at this point may be easily computed: it is definite positive, at least if $a$ is not too large (see [136]). The state (3.12) is thus a local minimizer which is not the global one. As argued in $[67,112,120]$, it therefore seems logical to look for local minimizers of the energy rather than global ones. In such a strategy, the question arises to know which local minimizer is the relevant one (recall that an energy functional of the type (2.13) may have incredibly many local minimizers). A natural choice among all local minimizers would be the long time limit of the solution of the dynamical problem. Such a dynamics is often too complicated to be integrated over long times in practice.

We describe in the following a possible alternative way to pick up a local minimizer. The approach is based on some dynamical model which is not the actual dynamics arising from the energy (2.13), but a gradient flow. We refer to [112] for the details, and give only the general flavor here. Consider the energy (3.3), in dimension 1, with only nearest-neighbor interaction, namely

$$
E_{\frac{1}{N}}(\varphi)=\frac{1}{N} \sum_{i=0}^{N-1}\left[W\left(\frac{\varphi\left(\frac{i+1}{N}\right)-\varphi\left(\frac{i}{N}\right)}{\frac{1}{N}}\right)-f\left(\frac{i}{N}\right) \varphi\left(\frac{i}{N}\right)\right] .
$$

The energy being only defined by $\varphi\left(\frac{i}{N}\right)$, for $i \in\{1,2, \ldots, N\}$, we may assume without loss of generality that $\varphi$ is affine in each interval $\left(\frac{i}{N}, \frac{i+1}{N}\right)$. In this space of piecewise affine functions, under some fairly general conditions on $E_{\frac{1}{N}}$, the problem

$$
\left\{\begin{array}{c}
\frac{\partial \varphi^{N}}{\partial t}=-E_{\frac{1}{N}}^{\prime}\left(\varphi^{N}\right) \\
\varphi^{N}(x, t=0)=\varphi_{0}^{N}(x)
\end{array}\right.
$$

may be shown to have a unique solution (see [112]). The initial condition $\varphi_{0}^{N}$ is any piecewise affine injective function. In addition, as $N$ goes to infinity, the solution of (3.21) converges to the solution of the continuous problem

$$
\left\{\begin{array}{l}
\frac{\partial \varphi}{\partial t}=-E^{\prime}(\varphi) \\
\varphi(x, t=0)=\varphi_{0}(x)
\end{array}\right.
$$

which is shown in [112] to exist and be unique. The limit energy $E$ is given by

$$
E(\varphi)=\int_{0}^{1} W\left(\varphi^{\prime}(x)\right) \mathrm{d} x-\int_{0}^{1} f(x) \varphi(x) \mathrm{d} x
$$

and $\varphi_{0}$ is the limit of the functions $\varphi_{0}^{N}$. It is next remarked that, if $\|f\|_{L^{1}}$ and $\left\|\varphi_{0}\right\|_{H^{1}}$ are small enough, the solution of (3.22) converges as $t$ goes to infinity to a local minimizer of the energy $E$, which does not depend on $\varphi_{0}$. Additionally, the energy $E(\varphi(t))$ is shown to be decreasing with respect to time along the trajectory $\varphi(t)$.

The analysis carried out in [112] assumes the $\lambda$-convexity of the energy $E$ (see [112] for the definition of this notion). The approach provides an interesting means to define the state of the material subject to the force $f$. Preliminary results investigate the dependance of this state upon the initial conditions $\varphi_{0}^{N}$. A point is that 
the dynamics (3.22) is highly dependent on the topology used to define the gradient flow. The long time limit deformation $\varphi$ is a local minimizer for a given topology, possibly not for another one. Besides, the numerical tests performed in [112] indicate that the method is well-suited for smooth deformations, which is consistent with the smallness condition on the forces, but that configurations with fractures are difficult to identify by this algorithm. Definite conclusions on the efficiency of the approach in such cases, which do occur when the material is subjected to large forces, are yet to be obtained.

Finally, a more natural way to compute local minimizers is to look for local minimizers in the vicinity of the minimizer at rest, namely the identity. This is the approach used in $[65,67]$, where it is shown that for sufficiently small forces and boundary conditions, there exists a local minimizer of the energy in a neighborhood of the reference configuration. Based on the implicit function theorem, this result has the advantage to be valid in any dimension, in contrast to the gradient flow theory of [112], which in its present state is valid only in dimension one.

\section{Conclusion}

We have reviewed in this article various works on atomistic to continuum limits and their relation to computational materials science. Despite the huge effort devoted to the subject, a lot remains to be done, both on the theoretical and on the numerical sides. Most continuum models are far from being completely analyzed. The relation of continuum theories with atomistic ones is not yet fully understood for physically relevant models. This is a motivation for further works, both on the mathematical analysis of these problems, and on their numerical analysis.

\section{REFERENCES}

[1] R. Alicandro and M. Cicalese, A general integral representation result for continuum limits of discrete energies with superlinear growth. SIAM J. Math. Anal. 36 (2004) 1-37.

[2] M. Anitescu, D. Negrut, P. Zapol and A. El-Azab, A note on the regularity of reduced models obtained by nonlocal quasicontinuum-like approach. Technical report ANL/MCS-P1303-1105, Argonne National Laboratory, Argonne, Illinois (2005). Available at http://www-unix.mcs.anl.gov/ anitescu/PUBLICATIONS/quasicont.pdf.

[3] N. Antonic, C.J. van Duijn, W. Jäger and A. Mikelic, Multiscale problems in science and technology. Challenges to mathematical analysis and perspectives. Springer (2002).

[4] M. Arndt and M. Griebel, Derivation of higher order gradient continuum models from atomistic models for crystalline solids. SIAM J. Multiscale Model. Simul. 4 (2005) 531-562.

[5] M. Arroyo and T. Belytshko, A finite deformation membrane based on inter-atomic potentials for the transverse mechanics of nanotubes. Mech. Mater. 35 (2003) 175-622.

[6] N.W. Ashcroft and N.D. Mermin, Solid-State Physics. Saunders College Publishing (1976).

[7] A. Askar, Lattice dynamical foundations of continuum theories. World Scientific, Philadelphia (1985).

[8] J.M. Ball, Convexity conditions and existence theorems in nonlinear elasticity. Arch. Rat. Mech. Anal. 63 (1977) $337-403$.

[9] J.M. Ball, Singularities and computation of miminizers for variational problems, in Foundations of Computational Mathematics, R. DeVore, A. Iserles and E. Suli Eds., Cambridge University Press London Mathematical Society Lecture Note Series 284 (2001) 1-20.

[10] J.M. Ball, Some open problems in elasticity, in Geometry, Mechanics, and Dynamics. Springer (2002) 3-59.

[11] J.M. Ball and R.D. James, Fine phase mixtures as minimizers of energy. Arch. Rat. Mech. Anal. 100 (1987) 13-52.

[12] J.M. Ball and R.D. James, Proposed experimental tests of a theory of fine microstructure and the two-well problem. Phil. Trans. Royal Soc. London A 338 (1992) 389-450.

[13] J.M. Ball and F. Murat, $W^{1, p}$-quasiconvexity and variational problems for multiple integrals. J. Funct. Anal. 58 (1984) $225-253$.

[14] T.J. Barth, T. Chan and R. Haimes Eds., Multiscale and multiresolution methods, Lecture notes in computational science and engineering 20. Springer (2002).

[15] P. Bénilan, H. Brezis and M. Crandall, A semilinear equation in $L^{1}\left(\mathbb{R}^{N}\right)$. Ann. Sc. Norm. Sup. Pisa 2 (1975) 523-555.

[16] A. Bensoussan, J.-L. Lions and G. Papnicolaou, Asymptotic analysis for periodic structures, Studies in Mathematics and its Applications 5. North-Holland (1978).

[17] F. Bethuel, G. Huisken, S. Müller and K. Steffen, Variational models for microstructures and phase transition, in Calculus of Variations and Geometric Evolution Problems, Lecture Notes in Mathematics 1713. Springer (1999) 85-210. 
[18] K. Bhattacharya, Microstructure of Martensite: Why It Forms and How It Gives Rise to the Shape-Memory Effect. Oxford Series on Materials Modelling, Oxford University Press (2003).

[19] K. Bhattacharya and G. Dolzmann, Relaxation of some multi-well problems. Proc. Royal Soc. Edinburgh A 131 (2001) 279-320.

[20] X. Blanc, A mathematical insight into ab initio simulations of solid phase, in Mathematical Models and Methods for Ab Initio Quantum Chemistry, M. Defranceschi and C. Le Bris Eds., Lect. Notes Chem. 74. Springer (2000) 133-158.

[21] X. Blanc, Geometry optimization for crystals in Thomas-Fermi type theories of solids. Comm. P.D.E. 26 (2001) 651-696.

[22] X. Blanc, Unique solvability for system of nonlinear elliptic PDEs arising in solid state physics. SIAM J. Math. Anal. 38 (2006) 1235-1248.

[23] X. Blanc and C. Le Bris, Optimisation de géométrie dans le cadre des théories de Thomas-Fermi pour les cristaux périodiques [Geometry optimization for Thomas-Fermi type theories of solids]. Note C.R. Acad. Sci. Sér. 1 329 (1999) 551-556.

[24] X. Blanc and C. Le Bris, Thomas-Fermi type models for polymers and thin films. Adv. Diff. Equ. 5 (2000) $977-1032$.

[25] X. Blanc and C. Le Bris, Periodicity of the infinite-volume ground-state of a one-dimensional quantum model. Nonlinear Anal., T.M.A 48 (2002) 791-803.

[26] X. Blanc and C. Le Bris, Définition d'énergies d'interfaces à partir de modèles atomiques. Note C.R. Acad. Sci. Sér. 1 340 (2005) 535-540.

[27] X. Blanc, C. Le Bris and F. Legoll, Analysis of a prototypical multiscale method coupling atomistic and continuum mechanics. ESAIM: M2AN 39 (2005) 797-826.

[28] X. Blanc, C. Le Bris and F. Legoll, Analysis of a prototypical multiscale method coupling atomistic and continuum mechanics: the convex case. Acta Math. Appl. Sinica (to appear).

[29] X. Blanc, C. Le Bris and P.-L. Lions, Convergence de modèles moléculaires vers des modèles de mécanique des milieux continus [From molecular models to continuum mechanics]. Note C.R. Acad. Sci. Sér. 1332 (2001) 949-956.

[30] X. Blanc, C. Le Bris and P.-L. Lions, From molecular models to continuum mechanics. Arch. Rat. Mech. Anal. 164 (2002) $341-381$.

[31] X. Blanc, C. Le Bris and P.-L. Lions, A definition of the ground state energy for systems composed of infinitely many particles. Comm. P.D.E 28 (2003) 439-475.

[32] X. Blanc, C. Le Bris and P.-L. Lions, Du discret au continu pour des modèles de réseaux aléatoires d'atomes [Discrete to continuum limit for some models of stochastic lattices of atoms]. Note C.R. Acad. Sci. Sér. 1. 342 (2006) 627-633.

[33] X. Blanc, C. Le Bris and P.-L. Lions, On the energy of some microscopic stochastic lattices. Arch. Rat. Mech. Anal. 184 (2007) 303-339.

[34] X. Blanc, C. Le Bris and P.-L. Lions (in preparation).

[35] A. Braides, $\Gamma$-convergence for beginners, Oxford Lecture Series in Mathematics and its Applications 22. Oxford University Press, Oxford (2002).

[36] A. Braides, Non-local variational limits of discrete systems. Commun. Contemp. Math. 2 (2000) $285-297$.

[37] A. Braides and M.S. Gelli, Continuum limits of discrete systems without convexity hypotheses. Math. Mech. Solids 7 (2002) 41-66.

[38] A. Braides and M.S. Gelli, Limits of discrete systems with long-range interactions. J. Convex Anal. 9 (2002) $363-399$.

[39] A. Braides and M.S. Gelli, The passage from discrete to continuous variational problems: a nonlinear homogenization process. Preprint of the Scuola Normale Superiore di Pisa (2003). Available at http://cvgmt.sns.it/cgi/get.cgi/papers/bragel03/

[40] A. Braides, G. Dal Maso and A. Garroni, Variational formulation of softening phenomena in fracture mechanics: the onedimensional case. Arch. Rat. Mech. Anal. 146 (1999) 23-58.

[41] A. Braides, M.S. Gelli and M. Sigalotti, The passage from nonconvex discrete systems to variational problems in Sobolev spaces: the one-dimensional case. Proc. Steklov Inst. Math. 236 (2002) 395-414.

[42] L. Breimana, Probability, Classics in Applied Mathematics. SIAM, Philadelphia (1992).

[43] H. Brezis, Semilinear equations in $\mathbb{R}^{N}$ without condition at infinity. Appl. Math. Optim. 12 (1984) $271-282$.

[44] V.V. Bulatov and T. Diaz de la Rubia, Multiscale modelling of materials. MRS Bulletin 26 (2001).

[45] D. Caillerie, A. Mourad and A. Raoult, Discrete homogenization in graphene sheet modeling, J. Elasticity 84 (2006) 33-68.

[46] C. Carstensen, Numerical Analysis of Microstructure, in Theory and Numerics of Differential Equations, J.F. Blowey, J.P. Coleman and A.W. Craig Eds., Springer (2001) 59-126.

[47] C. Carstensen and T. Roubíček, Numerical approximation of young measuresin non-convex variational problems. Numer. Math. 84 (2000) 395-415.

[48] I. Catto, C. Le Bris and P.-L. Lions, Limite thermodynamique pour des modèles de type Thomas-Fermi. Note C.R.A.S. Sér. 1322 (1996) 357-364.

[49] I. Catto, C. Le Bris and P.-L. Lions, Sur la limite thermodynamique pour des modèles de type Hartree et Hartree-Fock [On the thermodynamic limit for Hartree and Hartree-Fock type models]. Note C.R.A.S. Sér. 1 327 (1998) $259-266$.

[50] I. Catto, C. Le Bris and P.-L. Lions, Mathematical theory of thermodynamic limits: Thomas-Fermi type models. Oxford University Press (1998).

[51] I. Catto, C. Le Bris and P.-L. Lions, On the thermodynamic limit for Hartree-Fock type models. Ann. Inst. H. Poincaré, Anal. Non Linéaire 18 (2001) 687-760. 
[52] I. Catto, C. Le Bris and P.-L. Lions, On some periodic Hartree-type models for crystals. Ann. Inst. H. Poincaré, Anal. Non Linéaire 19 (2002) 143-190.

[53] I. Catto, C. Le Bris and P.-L. Lions, From atoms to crytals: a mathematical journey. Bull. Amer. Math. Soc. 42 (2005) 291-363.

[54] M. Chipot and D. Kinderlehrer, Equilibrium configurations of crystals. Arch. Rat. Mech. Anal. 103 (1988) $237-277$.

[55] P.G. Ciarlet, Mathematical elasticity, Vol. 1. North Holland (1993).

[56] G. Csányi, T. Albaret, G. Moras, M.C. Payne and A. De Vita, Multiscale hybrid simulation methods for material systems $J$. Phys. Condens. Matt. 17 (2005) R691.

[57] R. Dacorogna, Direct methods in the calculus of variations. Springer-Verlag Berlin (1989).

[58] G. Dal Maso, An introduction to $\Gamma$-convergence, Progress in Nonlinear Differential Equations and their Applications 8. Birkhäuser Boston, Inc., Boston, MA (1993).

[59] P. Deák, T. Frauenheim and M.R. Pederson, Eds., Computer simulation of materials at atomic level. Wiley (2000).

[60] B.N. Delaunay, N.P. Dolbilin, M.I. Shtogrin and R.V. Galiulin, A local criterion for regularity of a system of points. Sov. Math. Dokl. 17 (1976) 319-322.

[61] G. Dolzmann, Variational Methods for Crystalline Microstructure - Analysis and Computation. Springer-Verlag (2003).

[62] W. E and B. Engquist, The Heterogeneous Multi-Scale Methods. Comm. Math. Sci. 1 (2003) 87-132.

[63] W. E and Z. Huang, Matching conditions in atomistic-continuum modeling of materials. Phys. Rev. Lett. 87 (2001) 135501.

[64] W. E and Z. Huang, A dynamic atomistic-continuum method for the simulation of crystalline materials. J. Comp. Phys. 182 (2002) 234-261.

[65] W. E and P.B. Ming, Atomistic and continuum theory of solids, I. Preprint (2003).

[66] W. E and P.B. Ming, Analysis of multiscale methods. J. Comp. Math. 22 (2004) 210-219.

[67] W. E and P.B. Ming, Cauchy-Born rule and stability of crystals: static problems. Arch. Rat. Mech. Anal. 183 (2007) $241-297$.

[68] M. Fago, R.L. Hayes, E.A. Carter and M. Ortiz, Density-functional-theory-based local quasicontinuum method: Prediction of dislocation nucleation. Phys. Rev. B $\mathbf{7 0}$ (2004) 100102(R).

[69] I. Fonseca, Variational methods for elastic crystals. Arch. Rat. Mech. Anal. 97 (1987) 187-220.

[70] I. Fonseca, The lower quasiconvex envelope of the stored energy function for an elastic crystal. J. Math. Pures Appl. 67 (1988) $175-195$.

[71] G. Friesecke and R.D. James, A scheme for the passage from atomic to continuum theory for thin films, nanotubes and nanorods. J. Mech. Phys. Solids 48 (2000) 1519-1540.

[72] G. Friesecke, R.D. James and S. Müller, Rigorous derivation of nonlinear plate theory and geometric rigidity. C.R. Acad. Sci. Paris Sér. I 334 (2002) 173-178.

[73] G. Friesecke and F. Theil, Validity and failure of the Cauchy-Born hypothesis in a Two-Dimensional Mass-Spring Lattice. $J$. Nonlinear Sci. 12 (2002) 445-478.

[74] C.S. Gardner and C. Radin, The infinite-volume ground state of the Lennard-Jones potential. J. Stat. Phys. 20 (1979) 719-724.

[75] G. Geymonat, F. Krasucki and S. Lenci, Analyse asymptotique du comportement d'un assemblage collé [Asymptotic analysis of the behaviour of a bonded joint]. C.R. Acad. Sci. Paris Sér. I 322 (1996) 1107-1112.

[76] G. Geymonat, F. Krasucki and S. Lenci, Mathematical analysis of a bonded joint with a soft thin adhesive. Math. Mech. Solids 4 (1999) 201-225.

[77] WJ. Hehre, L. Radom, P.V.R. Shleyer and J. Pople, Ab initio molecular orbital theory. Wiley (1986).

[78] O. Iosifescu, C. Licht and G. Michaille, Variational limit of a one dimensional discrete and statistically homogeneous system of material points. Asymptot. Anal. 28 (2001) 309-329.

[79] O. Iosifescu, C. Licht and G. Michaille, Variational limit of a one-dimensional discrete and statistically homogeneous system of material points. C.R. Acad. Sci. Paris Sér. I Math. 332 (2001) 575-580.

[80] F. John, Rotation and strain. Comm. Pure Appl. Math. 14 (1961) 391-413.

[81] F. John, Bounds for deformations in terms of average strains, in Inequalities III, O. Shisha Ed. (1972) 129-144.

[82] D. Kinderlehrer, Remarks about equilibrium configurations of crystals, in Material instabilities in contiuum mechanics and related mathematical problems, J.M. Ball Ed., Oxford University Press (1998) 217-242.

[83] D. Kinderlehrer and P. Pedregal, Characterization of Young measures generated by gradients. Arch. Rat. Mech. Anal. 115 (1991) 329-365.

[84] D. Kinderlehrer and P. Pedregal, Gradient Young measures generated by sequences in Sobolev spaces. J. Geom. Anal. 4 (1994) 59-90.

[85] O. Kirchner, L.P. Kubin and V. Pontikis Eds., Computer simulation in materials science, Kluwer (1996).

[86] H. Kitagawa, T. Aihara Jr. and Y. Kawazoe Eds., Mesoscopic dynamics of fracture, Advances in Materials Research. Springer (1998).

[87] C. Kittel, Introduction to Solid State Physics. 7th edn. Wiley (1996).

[88] J. Knap and M. Ortiz, An Analysis of the QuasiContinuum Method. J. Mech. Phys. Solids 49 (2001) 1899.

[89] R.V. Kohn and G. Strang, Optimal design and relaxation of variational problems. I-II-III. Comm. Pure Appl. Math. 39 (1986) 113-137, 139-182, 353-377. 
[90] U. Krengel, Ergodic theorems, Studies in Mathematics 6. de Gruyter (1985).

[91] J. Kristensen, On the non-locality of quasiconvexity. Ann. Inst. H. Poincaré, Anal. Non Linéaire 16 (1999) 1-13.

[92] C. Le Bris, Computational Chemistry, in Handbook of numerical analysis, Vol. X, P.G. Ciarlet Ed., North-Holland (2003).

[93] C. Le Bris, Computational chemistry from the perspective of numerical analysis, Acta Numer. 14 (2005) 363-444.

[94] J. Li, K.J. Van Vliet, T. Zhu, S. Suresh and S. Yip, Atomistic mechanisms governing elastic limit and incipient plasticity in crystals. Nature 418 (2002) 307.

[95] C. Licht, Comportement asymptotique d'une bande dissipative mince de faible rigidité [Asymptotic behaviour of a thin dissipative layer with low stiffness]. C.R. Acad. Sci. Paris Sér. I 317 (1993) 429-433.

[96] C. Licht and G. Michaille, Une modélisation du comportement d'un joint collé élastique [A modelling of elastic adhesively bonding joints]. C.R. Acad. Sci. Paris Sér. I 322 (1996) 295-300.

[97] E.H. Lieb, Thomas-Fermi and related theories of atoms and molecules. Rev. Modern Phys. 53 (1981) 603-641 .

[98] E.H. Lieb and B. Simon, The Thomas-Fermi theory of atoms, molecules and solids. Adv. Math. 23 (1977) $22-116$.

[99] P. Lin, A nonlinear wave equation of mixed type for fracture dynamics. Research report No. 777, Department of Mathematics, The National University of Singapore, August 2000. Available at http://www.math.nus.edu.sg/ matlinp/WWW/linsiap.pdf

[100] P. Lin, Theoretical and numerical analysis of the quasi-continuum approximation of a material particle model. Math. Comput. 72 (2003) 657-675.

[101] P. Lin, Convergence analysis of a quasi-continuum approximation for a two-dimensional material. Preprint 2005-80 of the Institute for mathematical sciences, National University of Singapore (2005). Available at http://www.ims.nus.edu.sg/preprints/2005-80.pdf

[102] P. Lin and C.W. Shu, Numerical solution of a virtual internal bond model for material fracture. Physica D 167 (2002) 101-121.

[103] W.K. Liu, D. Qian and M.F. Horstemeyer, Special Issue on Multiple Scale Methods for Nanoscale Mechanics and Materials. Comp. Meth. Appl. Mech. Eng. 193 (2004) 17-20.

[104] M. Luskin, On the computation of crystalline microstructure. Acta Numer. 5 (1996) 191-258.

[105] M. Luskin, Computational modeling of microstructure, in Proceedings of the International Congress of Mathematicians, ICM, Beijing (2002) 707-716.

[106] R. Miller and E.B. Tadmor, The Quasicontinuum Method: Overview, applications and current directions. J. Computer-Aided Materials Design 9 (2002) 203-239.

[107] R. Miller, E.B. Tadmor, R. Phillips and M. Ortiz, Quasicontinuum simulation of fracture at the atomic scale. Modelling Simul. Mater. Sci. Eng. 6 (1998) 607.

[108] C.B. Morrey Jr., Quasi-convexity and the lower semi-continuity of multiple integrals. Pacific J. Math. 2 (1952) $25-53$.

[109] S. Müller, Variational models for microstructure and phase transitions, in Calculus of Variations and Geometric Evolution Problems. Lect. Notes Math. 1713. Springer Verlag, Berlin (1999) 85-210.

[110] B.R.A. Nijboer and W.J. Ventevogel, On the configuration of systems of interacting particles with minimum potential energy per particle. Physica 98A (1979) 274.

[111] B.R.A Nijboer and W.J. Ventevogel, On the configuration of systems of interacting particles with minimum potential energy per particle. Physica 99A (1979) 569 .

[112] C. Ortner, Continuum limit of a one-dimensional atomistic energy based on local minimization. Technical report 05/11, Oxford University Computing Laboratory (2005).

[113] S. Pagano and R. Paroni, A simple model for phase transitions: from the discrete to the continuum problem. Quart. Appl. Math. 61 (2003) 89-109.

[114] P. Pedregal, Parametrized Measures and Variational Principles. Birkhäuser (1997).

[115] P. Pedregal, Variational Methods in Nonlinear Elasticity. SIAM (2000).

[116] C. Pisani Ed., Quantum mechanical ab initio calculation of the properties of crystalline materials, Lecture Notes in Chemistry 67. Springer (1996).

[117] D. Raabe, Computational Material Science. Wiley (1998).

[118] C. Radin, Ground states for soft disks. J. Stat. Phys. 26 (1981) 365.

[119] Y.G. Reshetnyak, Liouville's theory on conformal mappings under minimal regularity assumptions. Sibirskii Math. 8 (1967) 69-85.

[120] M.O. Rieger and J. Zimmer, Young measure flow as a model for damage, SIAM J. Math. Anal. (2005) (to appear).

[121] R.E. Rudd and J.Q. Broughton, Concurrent coupling of length scales in solid state system, in [59] $251-291$.

[122] B. Schmidt, On the passage form atomic to continuum theory for thin films. Preprint 82/2005 of the Max Planck Institute of Leipzig (2005). Available at http://www.mis.mpg.de/preprints/2005/prepr2005_82.html

[123] B. Schmidt, Qualitative properties of a continuum theory for thin films. Preprint 83/2005 of the Max Planck Institute of Leipzig (2005). Available at http://www.mis.mpg.de/preprints/2005/prepr2005_83.html

[124] B. Schmidt, A derivation of continuum nonlinear plate theory form atomistic models. Preprint 90/2005 of the Max Planck Institute of Leipzig (2005). Available at http://www.mis.mpg.de/preprints/2005/prepr2005_90.html

[125] V.B. Shenoy, R. Miller, E.B. Tadmor, R. Phillips and M. Ortiz, Quasicontinuum models of interfacial structure and deformation. Phys. Rev. Lett. 80 (1998) 742. 
[126] V.B. Shenoy, R. Miller, E.B. Tadmor, D. Rodney, R. Phillips and M. Ortiz, An adaptative finite element approach to atomic-scale mechanics - the QuasiContinuum Method. J. Mech. Phys. Solids 47 (1999) 611.

[127] J.P. Solovej, Universality in the Thomas-Fermi-von Weizsäcker model of atoms and molecules. Comm. Math. Phys. 129 (1990) 561-598.

[128] V. Šveràk, On regularity for Monge-Ampère equations. Preprint, Heriott-Watt University (1991).

[129] V. Šveràk, Rank-one convexity does not imply quasiconvexity. Proc. Roy. Soc. Edinburgh A 120 (1992) $185-189$.

[130] V. Šveràk, On the problem of two wells, in Microstructure and phase transition, IMA Vol. Math. Appl. 54. Springer, New York, (1993) 183-189.

[131] A. Szabo and N.S. Ostlund, Modern quantum chemistry: an introduction. Macmillan (1982).

[132] E.B. Tadmor and R. Phillips, Mixed atomistic and continuum models of deformation in solids. Langmuir 12 (1996) 4529.

[133] E.B. Tadmor, M. Ortiz and R. Phillips, Quasicontinuum analysis of defects in solids. Phil. Mag. A. 73 (1996) $1529-1563$.

[134] E.B. Tadmor, G.S. Smith, N. Bernstein and E. Kaxiras, Mixed finite element and atomistic formulation for complex crystals. Phys. Rev. B 59 (1999) 235.

[135] F. Theil, A proof of crystallization in two dimensions. Comm. Math. Phys. 262 (2006) 209-236.

[136] L. Truskinovsky, Fracture as a phase transformation, in Contemp. Res. in Mech. and Math. of Materials, Ericksen's symposium, R. Batra and M. Beatty Eds., CIMNE, Barcelone (1996) 322-332.

[137] W.J. Ventevogel, On the configuration of a one-dimensional system of interacting particles with minimum potential energy per particle. Physica 92A (1978) 343.

[138] S. Yip, Synergistic materials science. Nature Mater. 2 (2003) 3-5.

[139] L.C. Young, Lectures on the calculus of variations and optimal control theory. W.B. Saunders Co., Philadelphia-LondonToronto (1969).

[140] F. Zaittouni, F. Lebon and C. Licht, Étude théorique et numérique du comportement d'un assemblage de plaques [Theoretical study of the behaviour of bonded plates]. C.R. Mécanique 330 (2002) 359-364. 